\title{
Distinguished material surfaces and coherent structures in three-dimensional fluid flows
}

\author{
G. Haller \\ Division of Applied Mathematics, Lefschetz Center for Dynamical Systems, Brown University, Providence, RI 02912, USA
}

Received 30 May 2000; received in revised form 18 September 2000; accepted 24 October 2000

Communicated by U. Frisch

\begin{abstract}
We prove analytic criteria for the existence of finite-time attracting and repelling material surfaces and lines in threedimensional unsteady flows. The longest lived such structures define coherent structures in a Lagrangian sense. Our existence criteria involve the invariants of the velocity gradient tensor along fluid trajectories. An alternative approach to coherent structures is shown to lead to their characterization as local maximizers of the largest finite-time Lyapunov exponent field computed directly from particle paths. Both approaches provide effective tools for extracting distinguished Lagrangian structures from three-dimensional velocity data. We illustrate the results on steady and unsteady ABC-type flows. (C) 2001 Elsevier Science B.V. All rights reserved.
\end{abstract}

Keywords: Fluid flows; Coherent structures; Invariant manifolds; Mixing

\section{Introduction}

The mixing of passive tracers in three-dimensional fluid flows is intimately linked to the presence of coherent structures. Without being specific about their definition for now, we shall picture these structures in the extended phase space of space and time, and refer to them as Lagrangian coherent structures. A fundamental question in the study of both turbulent and laminar mixing is the location and nature of these structures, as well as their interaction with each other.

For steady velocity fields, Lagrangian coherent structures are typically delineated by stable and unstable manifolds of invariant sets that are responsible for the stretching and folding of blobs of initial conditions. The "skeleton" formed by these manifolds has been recognized to have an important role in particle transport. Its existence in certain near-integrable cases has been studied numerically (see, e.g., [4,33,38]), analytically (see, e.g., [28,29,31]), and even experimentally (see [15]). Other structures, such as invariant tori or cylinders, are known to prevent mixing by providing closed two-dimensional invariant boundaries that trajectories cannot cross (see, e.g., [7,12,14,23]). If the velocity field is given analytically, one can locate hyperbolic fixed points and study their global influence through their stable and unstable manifolds (see, e.g., $[8,12,25,43])$. Some analytic techniques for locating stable and unstable manifolds also carry over to near-integrable three-dimensional flows with periodic time dependence

E-mail address: haller@cfm.brown.edu (G. Haller). 
(see, e.g., [24]). In such flows hyperbolic periodic orbits take over the role of hyperbolic stagnation points as basic organizing centers for mixing. They can be targeted with special numerical methods even in flows that are far from integrable (see, e.g., [2,27]). Finally, long-term statistical properties of material stretching (associated with hyperbolic foliations) have been analyzed in several studies of three-dimensional steady or time-periodic flows (see, e.g., [18]).

With the advent of computational fluid dynamics, there is an increasing need to understand and evaluate mixing in numerical velocity data with general (turbulent) time dependence. These data sets are discrete and hence velocities are not available at all points. This fact makes it numerically challenging to locate even fixed points, let alone more general dynamical structures. The main difficulty is, however, the fact the velocity data is only available on a finite-time interval. Unlike for flows with periodic or quasiperiodic time dependence, the asymptotic behavior of particles now cannot be recovered from a finite-time velocity sample. As a result, Poincaré maps are no longer available, classical stable and unstable manifolds become undefined, and the tools to locate them either fail or provide results that are difficult to interpret. At the same time, statistics-based approaches, such as long-term plots of passive scalar gradient fields, do suggest the presence of distinct Lagrangian coherent structures that resemble stable and unstable manifolds (see, e.g., [16]).

The same problem already arises in the study of two-dimensional turbulent flows. A large body of literature has been developed on the statistical evaluation of Lagrangian dynamics from two-dimensional velocity data (see [37] for a survey). More recently, several numerical studies have been aimed at understanding the geometry of Lagrangian coherent structures in specific two-dimensional data sets (see, e.g., [9,30,35,34]). A theoretical approach to finite-time invariant manifolds (material lines) has been developed in [19-21]. It involves simple analytic conditions on the invariants of the velocity gradient tensor along particles paths. The conditions can be used to associate a time to each fluid particle during which it travels in a linearly stable or unstable material line. Lagrangian coherent structures are then identified as local minimizers or maximizers of the stability or instability time field. This result provides a connection between Eulerian approaches to coherent structures, notably the Okubo-Weiss criterion (see, e.g., [41]), and actual Lagrangian structures.

In the present paper, we extend the two-dimensional analysis of Haller [20] to three-dimensional velocity fields of the form $\mathbf{u}(\mathbf{x}, t), t \in I$, where $I$ is a finite time interval. Our goal is to extract dynamically important material lines and surfaces from a data set representing $\mathbf{u}$. As in the two-dimensional case, we want to achieve this by deriving conditions under which sample particle paths $\mathbf{x}(t)$ generated by $\mathbf{u}$ lie on finite-time hyperbolic material lines or surfaces (to be defined below) on some subset of the time interval $I$. The conditions turn out to involve the eigenvalues of $\nabla \mathbf{u}(\mathbf{x}(t), t)$, as well as an invariant $\beta$ of $\nabla \mathbf{u}(\mathbf{x}(t), t)$ that measures how fast its eigenvectors change in time. Lagrangian coherent structures will then be identified as material lines or surfaces along which our conditions hold for locally the longest time in the flow. A sample result of such an analysis is shown in Fig. 1, which was obtained from a finite-time stability analysis of the well-known ABC flow (see Section 5 for details and notation).

The extension we complete here from two- to three-dimensional flows is technically challenging and requires customized treatments of the different eigenvalue configurations of $\nabla \mathbf{u}(\mathbf{x}(t), t)$. Accordingly, the resulting attracting and repelling material surfaces and lines fall into ten different categories (cf. Fig. 3), as opposed to two categories in the two-dimensional case. Surprisingly, the formulae obtained in this fashion are still simple, but their numerical implementation requires more thought than in two dimensions.

To compare our analytic results on distinguished Lagrangian structures with actual "bulk" particle dynamics, we use an alternative, purely geometric approach. Again, we seek material lines and surfaces that repel or attract infinitesimally close particles for locally the longest time. However, we now approach such structures by searching for material lines and surfaces transverse to which unit vectors grow by the largest amount over the time interval considered. As we show, such structures are local maximizers of the largest finite-time Lyapunov exponent field, computed directly from trajectories. This second approach to locating Lagrangian coherent structures converges 


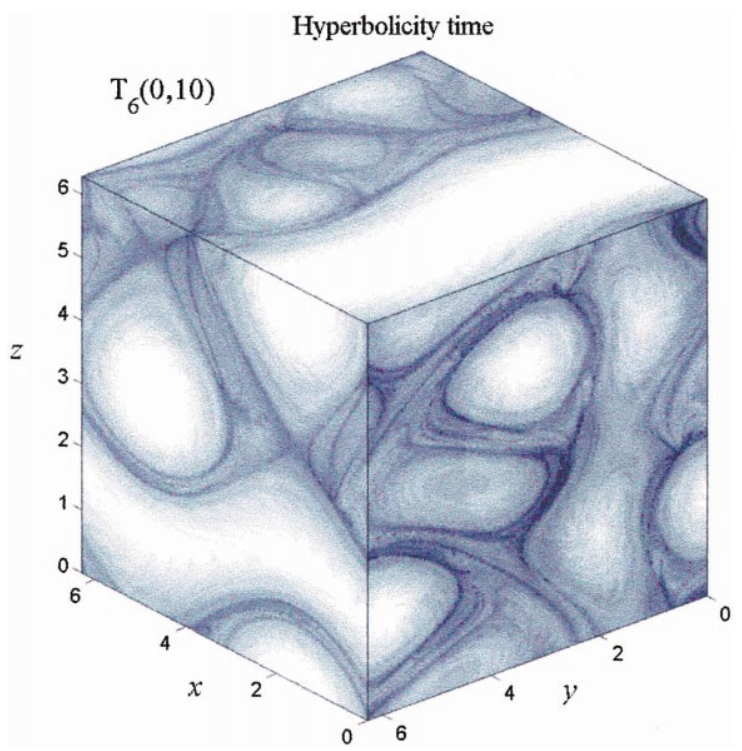

Fig. 1. Finite-time hyperbolicity times for the $\mathrm{ABC}$ flow with $A=\sqrt{3}, B=\sqrt{2}$, and $C=1$, on the time interval $[0,10]$. Darker colors indicate longer times. Local maxima in the plot are repelling Lagrangian coherent structures, responsible for the stretching of a generic blob of fluid.

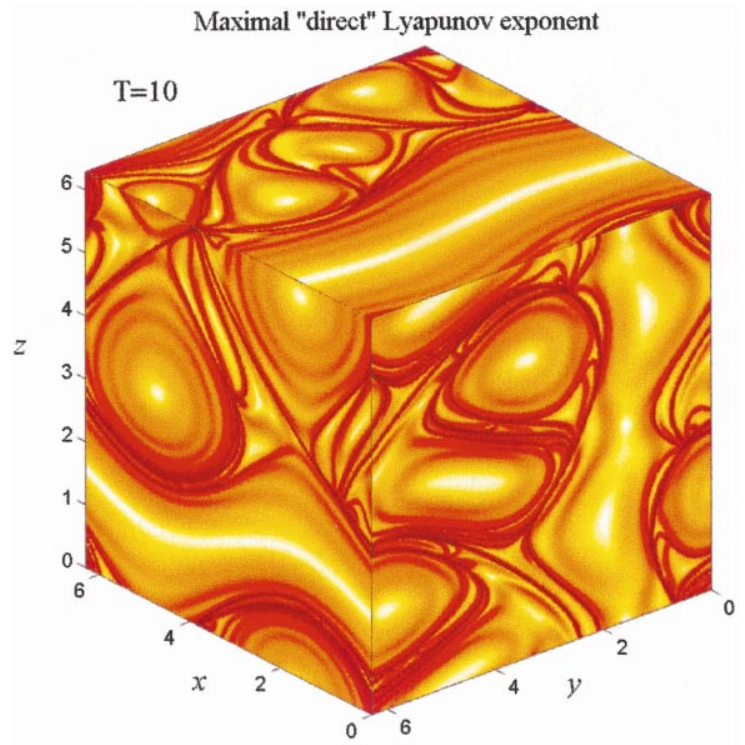

Fig. 2. Maximal "direct" finite-time Lyapunov exponent for the ABC flow, computed by differentiating trajectory positions at $T=10$ with respect to their initial conditions at $t=0$. Darker colors indicate larger values. Local maxima indicate repelling coherent structures. 
more slowly initially, but provides well-resolved images for long-lived structures. While the direct connection between these images and actual structures is lost for longer times, on intermediate time scales they do offer a good comparison with our analytic results. This can be seen by comparing Figs. 1 and 2 .

This paper is organized as follows. In Section 2, we define attracting and repelling material lines and surfaces for three-dimensional finite-time velocity fields. We first give a hyperbolicity time definition of coherent structures and then describe our alternative geometric approach for locating them numerically from available particle data. In Section 3, we make some basic assumptions on the velocity field, then identify different eigenvalue configurations of interest for the matrix $\nabla \mathbf{u}(\mathbf{x}(t), t)$. We then proceed to the statement of Theorem 1, our main result, on the existence of attracting and repelling material lines and surfaces. A detailed proof of this theorem is given in Appendix A. Section 4 is devoted to the numerical implementation of the main theorem and Section 5 describes our numerical experiments with steady and forced ABC flows. Section 6 offers conclusions and outlines some open questions.

\section{Finite-time coherent structures}

\subsection{Basic concepts: finite-time hyperbolic material surfaces and lines}

Consider a three-dimensional velocity field

$$
\dot{\mathbf{x}}=\mathbf{u}(\mathbf{x}, t), \quad \mathbf{x} \in \mathbb{R}^{3}, \quad t \in I,
$$

where $\mathbf{u}$ is a continuously differentiable function of its variables, and $I$ a finite-time interval. A trajectory generated by this velocity will be denoted by $\mathbf{x}\left(t ; \bar{t}, \mathbf{x}_{0}\right)$, where $\bar{t}$ refers to the time when the trajectory is at the initial position $\mathbf{x}_{0}$. When unimportant, we shall omit the explicit reference to $\bar{t}$ and $\mathbf{x}_{0}$ in our notation and simply write $\mathbf{x}(t)$.

We recall that a material line $\mathcal{L}(t)$ is a smooth curve of fluid particles advected by the velocity field. Similarly, a material surface $\mathcal{M}(t)$ is a smooth, evolving surface of particles. Let $\mathcal{I}$ be an open time interval within $I$. We will call a material surface or line attracting over $\mathcal{I}$, if it is linearly stable for times taken from $\mathcal{I}$. More concretely, infinitesimal perturbations from $\mathcal{L}(t)$ and $\mathcal{M}(t)$ should result in exponential convergence to these material objects while $t \in \mathcal{I}$. Similarly, we shall call a material surface or line repelling over $\mathcal{I}$, if it is attracting over $\mathcal{I}$ in backward time. This requires infinitesimally close fluid particles to separate from these objects at an exponential rate while $t \in \mathcal{I}$. ${ }^{1}$ Finally, we shall call a material surface or line finite-time hyperbolic over $\mathcal{I}$, if it is attracting or repelling over $\mathcal{I}$ in the sense described above. ${ }^{2}$

In Fig. 3, we illustrate the Lagrangian dynamics near different types of finite-time hyperbolic material lines and surfaces. The figure only details geometries found in nearly incompressible flows. It is important to note that Fig. 3, while depicts snapshots, does not merely show instantaneous Lagrangian dynamics. The surfaces and lines containing $\mathbf{x}(t)$ continue to attract or repel particles over the whole time interval $\mathcal{I}$. In other words, their existence is by no means obvious from the eigenvalue configuration of the velocity gradient $\nabla \mathbf{u}$ at $(\mathbf{x}(t), t)$. While $\nabla \mathbf{u}$ and the rate of strain tensor $\mathbf{D}=\frac{1}{2}\left(\nabla \mathbf{u}+\nabla \mathbf{u}^{\mathrm{T}}\right)$ have been extensively studied in the literature, their instantaneous stretching or compressing properties by themselves have no direct relevance to true Lagrangian dynamics over an extended period of time.

\footnotetext{
${ }^{1}$ If the length of $\mathcal{I}$ tends to infinity, attracting material lines and surfaces become what we traditionally call unstable manifolds in infinite-time dynamical systems, at least for nearly incompressible flows. The reason is that volume preservation requires any exponential separation or convergence transverse to a material object to be balanced by an opposite trend on the object itself.

${ }^{2}$ For mathematically more precise definitions, the reader is referred to Appendix A, where our main result is proved.
} 


\section{Finite-time hyperbolic Finite-time hyperbolic material lines material surfaces}
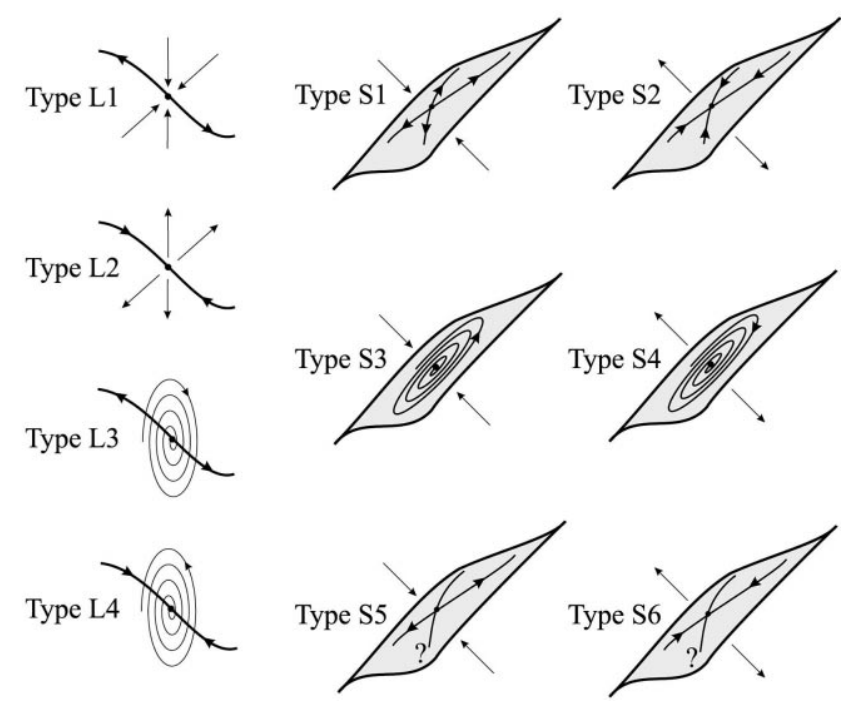

Fig. 3. Different types of finite-time hyperbolic material lines and surfaces containing a trajectory $\mathbf{x}(t)$. The figures show instantaneous positions of these lines and surfaces at time $t$ with $\mathbf{x}(t)$ appearing as a single point.

\subsection{Lagrangian coherent structures: an analytic approach}

Using finite-time hyperbolic material lines and surfaces, we can give a possible description of Lagrangian coherent structures. The approach we take in this section will later enable us to describe these structures in terms of the invariants of the velocity gradient tensor.

A general material line or surface will change its stability type many times, as it is influenced by different coherent structures it passes by. However, a Lagrangian coherent structures, denoted by $\mathcal{C}(t)$, is distinguished in one of the following two ways:

1. $\mathcal{C}(t)$ retains its stability type (i.e., the type of impact it has on neighboring orbits) for locally the longest time in the flow.

2. $\mathcal{C}(t)$ retains its stability type for locally the shortest time in the flow.

While case (1) includes all generic coherent structures in an open flow, case (2) is relevant for flows with a no-slip boundary. In this case some coherent structures, such as those near separation or reattachment points, may be attached to the wall. Right where they attach to the wall, these structures fail to be finite-time hyperbolic for any length of time, since the linearized flow is degenerate along them. Accordingly, parts of these structures close to the wall can only be finite-time hyperbolic for short times. At the same time, nearby initial conditions simply pass by the wall and will, generically, accumulate hyperbolicity times throughout their later history. For this reason, $\mathcal{C}(t)$ will exhibit finite-time hyperbolicity for locally the shortest time in a vicinity of the wall. Both cases (1) and (2) occur already in two-dimensional turbulence (see [21] for more discussion).

Motivated by the above observations, consider an initial condition $\mathbf{x}_{0}$, and define the scalar functions $T_{\mathbf{L N}}\left(\mathbf{x}_{0} ; \bar{t}, t\right)$, $\mathbf{N}=1, \ldots, 4$, as the total length of time within $[\bar{t}, t]$ over which the trajectory $\mathbf{x}\left(t ; \bar{t}, \mathbf{x}_{0}\right)$ lies in a material line of 
stability type $\mathbf{L N}$ (cf. Fig. 3). Similarly, define the scalar functions $T_{\mathbf{S M}}\left(\mathbf{x}_{0} ; \bar{t}, t\right), \mathbf{M}=1, \ldots, 6$, as the total length of time within $[\bar{t}, t]$ over which the trajectory $\mathbf{x}\left(t ; \bar{t}, \mathbf{x}_{0}\right)$ lies in a material surface of stability type $\mathbf{S M}$. We introduce the following defining principle for Lagrangian coherent structures:

Coherent structures are characterized by local extrema in the scalar fields $T_{\mathbf{L M}}\left(\mathbf{x}_{0} ; \bar{t}, t\right)$ and/or $T_{\mathbf{S N}}\left(\mathbf{x}_{0} ; \bar{t}, t\right)$ for some $\mathbf{M}$ and $\mathbf{N}$.

Once one adapts the above description of Lagrangian coherent structures, the main technical issue in their detection becomes the computation of the scalar fields $T_{\mathbf{L M}}\left(\mathbf{x}_{0} ; \bar{t}, t\right)$ and $T_{\mathbf{S N}}\left(\mathbf{x}_{0} ; \bar{t}, t\right)$. In principle, this is a hopeless undertaking since one would have to verify the stability of all possible material surfaces and lines in the flow. Our main mathematical result in Section 3.2 is precisely about circumventing this problem: we shall be able to compute the fields $T_{\mathbf{L M}}\left(\mathbf{x}_{0} ; \bar{t}, t\right)$ and $T_{\mathbf{S N}}\left(\mathbf{x}_{0} ; \bar{t}, t\right)$ in a very simple way, using only the invariants on the velocity gradient along the trajectory $\mathbf{x}\left(t ; \bar{t}, \mathbf{x}_{0}\right)$.

\subsection{Lagrangian coherent structures: a geometric approach}

We now discuss an alternative approach to Lagrangian coherent structures. It is primarily geared towards extracting such structures from available particle paths without using the velocity field that generated the trajectories.

In agreement with the previous section, we view coherent structures as linearly stable or unstable material lines or surfaces. If they attract or repel particles for locally the longest time in the flow, the maximal net growth of a unit vector transverse to them should be locally the largest over the time interval of interest. Consider, for instance, a repelling structure $\mathcal{C}(t)$, and select a point $\mathbf{x}_{0} \in \mathcal{C}(\bar{t})$. We also select a unit vector $\mathbf{e}_{\bar{t}}$ at $\mathbf{x}_{0}$ which is not tangent to $\mathcal{C}(\bar{t})$. We propagate $\mathbf{e}_{\bar{t}}$ along the trajectory $\mathbf{x}\left(t, \bar{t}, \mathbf{x}_{0}\right)$ using the linearized flow map $\nabla_{\mathbf{x}_{0}} \mathbf{x}\left(t, \bar{t}, \mathbf{x}_{0}\right)$ to obtain the vector

$$
\mathbf{e}_{t}\left(\mathbf{x}_{0}\right)=\nabla_{\mathbf{x}_{0}} \mathbf{x}\left(t, \bar{t}, \mathbf{x}_{0}\right) \mathbf{e}_{\bar{t}} .
$$

In order to locate repelling coherent structures, we now seek to maximize $\left|\mathbf{e}_{t}\left(\mathbf{x}_{0}\right)\right|$ over all possible choices of $\mathbf{e}_{\bar{t}}$ and $\mathbf{x}_{0}$. Using the operator norm

$$
\|\mathbf{A}\|=\max _{|\mathbf{x}|=1}|\mathbf{A x}|
$$

for a general matrix $\mathbf{A} \in \mathbb{R}^{3}$, we can immediately maximize $\left|\mathbf{e}_{t}\left(\mathbf{x}_{0}\right)\right|$ over all choices of $\mathbf{e}_{\bar{t}}$ :

$$
\mathcal{E}_{\bar{t}}^{t}\left(\mathbf{x}_{0}\right)=\max _{|\hat{\mathbf{e}}|=1}\left|\nabla_{\mathbf{x}_{0}} \mathbf{x}\left(t, \bar{t}, \mathbf{x}_{0}\right) \mathbf{e}_{\bar{t}}\right|=\left\|\nabla_{\mathbf{x}_{0}} \mathbf{x}\left(t, \bar{t}, \mathbf{x}_{0}\right)\right\| .
$$

Now recall that for any matrix $\mathbf{A},\|\mathbf{A}\|$ is actually equal to the square root of the maximal eigenvalue of the positive definite symmetric matrix $\mathbf{A}^{\mathrm{T}} \mathbf{A}$, which we denote by $\lambda_{\max }\left(\mathbf{A}^{\mathrm{T}} \mathbf{A}\right)$. We can therefore write

$$
\mathcal{E}_{\bar{t}}^{t}\left(\mathbf{x}_{0}\right)=\sqrt{\lambda_{\max }\left(\nabla_{\mathbf{x}_{0}} \mathbf{x}\left(t, \bar{t}, \mathbf{x}_{0}\right)^{\mathrm{T}} \nabla_{\mathbf{x}_{0}} \mathbf{x}\left(t, \bar{t}, \mathbf{x}_{0}\right)\right)} .
$$

We also recall that, by definition, the largest finite-time Lyapunov exponent, $\Lambda\left(t, \bar{t}, \mathbf{x}_{0}\right)$, associated with the trajectory $\mathbf{x}\left(t, \bar{t}, \mathbf{x}_{0}\right)$ is just

$$
\Lambda\left(t, \bar{t}, \mathbf{x}_{0}\right)=\log \left[\lambda_{\max }\left(\nabla_{\mathbf{x}_{0}} \mathbf{x}\left(t, \bar{t}, \mathbf{x}_{0}\right)^{\mathrm{T}} \nabla_{\mathbf{x}_{0}} \mathbf{x}\left(t, \bar{t}, \mathbf{x}_{0}\right)\right)^{1 / 2(t-\bar{t})}\right],
$$

thus we can rewrite $\mathcal{E}_{\bar{t}}^{t}\left(\mathbf{x}_{0}\right)$ as

$$
\mathcal{E}_{\bar{t}}^{t}\left(\mathbf{x}_{0}\right)=\mathrm{e}^{\Lambda\left(t, \bar{t}, \mathbf{x}_{0}\right)(t-\bar{t})} .
$$


The local maxima of $\mathcal{E}_{\bar{t}}^{t}\left(\mathbf{x}_{0}\right)$ therefore coincide with the local maxima of $\Lambda\left(t, \bar{t}, \mathbf{x}_{0}\right)$. Our alternative, velocityindependent principle for locating Lagrangian coherent structures can therefore be summarized as follows:

Coherent structures are characterized by local extrema of the scalar field $\Lambda\left(t, \bar{t}, \mathbf{x}_{0}\right)$ in forward or backward time.

In general, computing the quantity $\Lambda\left(t, \bar{t}, \mathbf{x}_{0}\right)$ directly from its definition (3) (i.e., taking the numerical derivative of particle positions at time $t$ with respect to a grid of initial positions) does not yield the true largest Lyapunov exponent; that is why such direct calculation is usually not attempted in the literature (see, e.g., [1] for a survey of existing methods). The reason is that the function $\nabla_{\mathbf{x}_{0}} \mathbf{x}\left(t, \bar{t}, \mathbf{x}_{0}\right)$ grows exponentially in time for typical initial conditions, and hence discretized approximations to it produce growing errors [42]. However, our main interest is finite-time phenomena here, in which case, for finite $t, \nabla_{\mathbf{x}_{0}} \mathbf{x}\left(t, \bar{t}, \mathbf{x}_{0}\right)$ can be computed with reasonable precision from a dense enough grid of $\mathbf{x}_{0}$ values. This follows from the smooth dependence of a trajectory on its initial condition. In backward time (i.e., for $\bar{t}>t$ ), the local maxima of $\Lambda\left(t, \bar{t}, \mathbf{x}_{0}\right)$ indicate attracting coherent structures. We note that the local maximization property of (infinite-time) stable manifolds for maximal finite-time Lyapunov exponents, computed along trajectories in the usual way, was already observed and used in [11].

We shall use $\Lambda\left(t, \bar{t}, \mathbf{x}_{0}\right)$ as defined in (3) to test the practical use of our hyperbolicity time approach. As we shall see in Section 5 , the convergence of maxima of $\Lambda\left(t, \bar{t}, \mathbf{x}_{0}\right)$ to coherent structures is initially slower than that of the fields $T_{\mathbf{L M}}\left(\mathbf{x}_{0} ; \bar{t}, t\right)$ and $T_{\mathbf{S N}}\left(\mathbf{x}_{0} ; \bar{t}, t\right)$. Later in time, however, the fact that it is computed solely based on trajectories, without using $\nabla \mathbf{u}$, leads to sharp maxima. This is due to the fact that when computed directly from formula (3) over an initial grid of particles, $\Lambda\left(t, \bar{t}, \mathbf{x}_{0}\right)$ is free from inconsistencies between the velocity field and actual computed trajectories. This inconsistency is the result of numerical errors, which are significant in chaotic flow fields like those we study in Section 5. Over long times, local extrema of $\Lambda\left(t, \bar{t}, \mathbf{x}_{0}\right)$ may not converge to true coherent structures associated with the velocity field, but they will converge to structures that are consistent with the available approximate particle data.

\section{An analytic result on coherent structures}

In this section, we describe how hyperbolicity times can be calculated for trajectories generated by the velocity field $\mathbf{u}(\mathbf{x}, t)$. The main theorem listed here will enable us to locate coherent structures obeying the definition we gave in Section 2.2.

\subsection{Assumptions and notation}

Let us consider a trajectory $\mathbf{x}(t)$ of the velocity field (1). The velocity gradient along $\mathbf{x}(t)$ is given by the time-dependent matrix $\nabla \mathbf{u}(\mathbf{x}(t), t)$. We denote the eigenvalues of this time-dependent matrix by $\lambda_{1}(t), \lambda_{2}(t)$ and $\lambda_{3}(t)$, ordered such that $\operatorname{Re} \lambda_{1} \leq \operatorname{Re} \lambda_{2} \leq \operatorname{Re} \lambda_{3}$. For any given $t$, at least one of these eigenvalues is real. We shall assume that the flow is neither strongly compressive nor strongly expanding, by which we mean that for any $t \in I$,

$$
\operatorname{Re} \lambda_{1}(t)<0<\operatorname{Re} \lambda_{3}(t) .
$$

This assumption is not crucial, but limits the number of possible cases considered in our analysis to those feasible in fluid mechanics. We shall be interested in studying the velocity field (1) on a closed time interval $\mathcal{I} \subset I$, on which one of the following five conditions is satisfied for $j=1,2,3$ : 
(A1)

$\operatorname{Im} \lambda_{j}(t) \equiv 0, \quad \lambda_{1}(t)<\lambda_{2}(t)<0<\lambda_{3}(t),\left.\quad \stackrel{\lambda_{1} \lambda_{2}}{\lambda^{\operatorname{Im} \lambda}}\right|_{\operatorname{Re\lambda }} ^{\lambda_{3}}$

$\operatorname{Im} \lambda_{j}(t) \equiv 0, \quad \lambda_{1}(t)<0<\lambda_{2}(t)<\lambda_{3}(t), \quad \stackrel{\lambda_{1}}{\left.\right|^{\operatorname{Im} \lambda}}{\stackrel{\lambda_{2}}{\lambda_{3}}}_{\text {Rei }}$

$\operatorname{Im} \dot{\lambda}_{j}(t) \equiv 0, \quad \lambda_{1}(t)<\lambda_{2}(t)<\lambda_{3}(t), \quad \stackrel{\left.\lambda_{1} \lambda_{2}\right|^{\operatorname{Im} \lambda} \overbrace{}^{\lambda_{3}}}{\longrightarrow} \operatorname{Re\lambda }$

$\operatorname{Im} \lambda_{1,2}(t)=\omega(t) \neq 0, \quad \operatorname{Re} \lambda_{1}(t)=\operatorname{Re} \lambda_{2}(t)<0<\lambda_{3}(t), \quad \stackrel{\left.\lambda_{1} \cdot\right|_{\lambda_{2}^{*}} ^{\operatorname{Im} \lambda}{ }^{\lambda_{3}}}{\operatorname{Re} \lambda}$

$\operatorname{Im} \lambda_{2,3}(t)=\omega(t) \neq 0, \quad \lambda_{1}(t)<0<\operatorname{Re} \lambda_{2}(t)=\operatorname{Re} \lambda_{3}(t), \quad{\frac{\lambda_{1}}{\operatorname{Im} \lambda}}_{\dot{\lambda}_{2} \lambda_{3}}^{\operatorname{Re} \lambda}$

Note that with the exception of (A3), all the above conditions require the real parts of the eigenvalues to have constant signs over the interval $\mathcal{I}$. In the case of (A3), $\lambda_{2}(t)$ is allowed to vary between the negative eigenvalue $\lambda_{1}(t)$ and the positive eigenvalue $\lambda_{3}(t)$ (cf. assumption (4)). For all cases listed in (A1)-(A5), we shall use the notation

$$
\lambda_{j \min }=\min _{t \in \mathcal{I}}\left|\operatorname{Re} \lambda_{j}(t)\right|
$$

Let us define the nonsingular $3 \times 3$ matrix

$$
\mathbf{M}=\left[\mathbf{e}_{1}(t), \mathbf{e}_{2}(t), \mathbf{e}_{3}(t)\right],
$$

where, for the cases (A1)-(A3), $\mathbf{e}_{j}(t)$ denotes a unit eigenvector of $\nabla \mathbf{u}(\mathbf{x}(t), t)$ corresponding to the eigenvalue $\lambda_{j}(t)$. For cases (A4) and (A5), $\mathbf{e}_{1}(t)$ and $\mathbf{e}_{2}(t)$ are defined as the real and imaginary parts (normalized to 1$)$ of the complex eigenvector corresponding to $\lambda_{1,2}(t)$, and $\mathbf{e}_{3}(t)$ denotes the unit eigenvector associated 
with $\lambda_{3}(t)$. In all cases, $\mathbf{e}_{j}(t)$ can be selected as a smooth function of time. We shall also use the quantity (cf. Section 2.3 for notation)

$$
\beta=\max _{t \in \mathcal{I}}\left\|\mathbf{M}^{-1}(t) \dot{\mathbf{M}}(t)\right\|=\max _{t \in \mathcal{I}} \sqrt{\lambda_{\max }\left([\dot{\mathbf{M}}(t)]^{\mathrm{T}}\left[\mathbf{M}^{-1}(t)\right]^{\mathrm{T}} \mathbf{M}^{-1}(t) \dot{\mathbf{M}}(t)\right)} .
$$

Note that $\beta$ is an invariant of the matrix $\nabla \mathbf{u}(\mathbf{x}(t), t)$ over the time interval $\mathcal{I}$. In fact, the matrix $\mathbf{M}^{-1}(t) \dot{\mathbf{M}}(t)$ itself is invariant with respect to time-independent changes of basis. As we shall see below, the invariant $\beta$ has a crucial role in identifying finite-time hyperbolic material lines and surfaces.

\subsection{The main theorem: existence of finite-time hyperbolic structures}

We now give conditions under which the trajectory $\mathbf{x}(t)$ lies on a finite-time hyperbolic material surface or material line throughout the time interval $\mathcal{I}$. In the statements below we shall refer to different material stability types as they are labeled in Fig. 3.

\section{Theorem 1.}

1. Suppose that (A1) (or (A4)) holds and

$$
\frac{\beta}{\lambda_{2 \min }}+\sqrt{\frac{\beta}{\lambda_{3 \min }}}<1 .
$$

Then $\mathbf{x}(t)$ is contained in an attracting material line of type $\mathrm{L} 1$ (or L3, resp.) for $t \in \mathcal{I}$.

2. Suppose that (A1) (or (A4)) holds and

$$
\sqrt{\frac{\beta}{\lambda_{2 \min }}}+\frac{\beta}{\lambda_{3 \min }}<1
$$

Then $\mathbf{x}(t)$ is contained in a repelling material surface of type $\mathrm{S} 2$ (or $\mathrm{S} 4$, resp.) for $t \in \mathcal{I}$.

3. Suppose that (A2) (or (A5)) holds and

$$
\frac{\beta}{\lambda_{2 \min }}+\sqrt{\frac{\beta}{\lambda_{1 \min }}}<1 .
$$

Then $\mathbf{x}(t)$ is contained in a repelling material line of type $\mathrm{L} 2$ (or $\mathrm{L} 4$, resp.) for $t \in \mathcal{I}$.

4. Suppose that (A2) (or (A5)) holds and

$$
\sqrt{\frac{\beta}{\lambda_{2 \min }}}+\frac{\beta}{\lambda_{1 \min }}<1 .
$$

Then $\mathbf{x}(t)$ is contained in an attracting material surface of type $\mathrm{S} 1$ (or $\mathrm{S} 3$, resp.) for $t \in \mathcal{I}$.

5. Suppose that (A3) holds and $\max _{t \in \mathcal{I}} \lambda_{2}(t)=\lambda_{2 \max }^{+} \geq 0$. Assume that

$$
\lambda_{3 \min }>\frac{2 \beta\left(\lambda_{2 \max }^{+}+2 \beta\right)}{\lambda_{2 \max }^{+}+5 \beta-\sqrt{\left(\lambda_{2 \max }^{+}+5 \beta\right)^{2}-4 \beta\left(\lambda_{2 \max }^{+}+2 \beta\right)}} .
$$

Then $\mathbf{x}(t)$ is contained in a repelling material surface of type S6 for $t \in \mathcal{I}$. 
6. Suppose that (A3) holds and $\max _{t \in \mathcal{I}}\left(-\lambda_{2}(t)\right)=\lambda_{2 \max }^{-} \geq 0$. Assume that

$$
\lambda_{1 \min }>\frac{2 \beta\left(\lambda_{2 \max }^{-}+2 \beta\right)}{\lambda_{2 \max }^{-}+5 \beta-\sqrt{\left(\lambda_{2 \max }^{-}+5 \beta\right)^{2}-4 \beta\left(\lambda_{2 \max }^{-}+2 \beta\right)}} .
$$

Then $\mathbf{x}(t)$ is contained in an attracting material surface of type S5 for $t \in \mathcal{I}$.

\subsection{Remarks on the main theorem}

1. Physical meaning. Physically speaking, the theorem requires typical Lagrangian velocities near a finite-time hyperbolic material object to dominate the "Eulerian deformation rate" due to the rotation of eigenvectors of the velocity gradient along particle paths.

2. Galilean invariance. The conditions of the theorem will be satisfied for the same material line or surface even after a change of coordinates $\mathbf{x}^{\prime}=\mathbf{Q x}+\mathbf{v}_{0} t$, where $\mathbf{Q}$ is a proper orthogonal $3 \times 3$ matrix and $\mathbf{v}_{0}$ a constant velocity. In fact, the results are invariant under the more general set of transformations $\mathbf{x}^{\prime}=\mathbf{Q x}+\mathbf{v}(t)$, where $\mathbf{v}(t)$ is an arbitrary function of time. A consequence of Galilean invariance is the independence of our criteria from the presence of instantaneous stagnation points, a feature that is currently used throughout the literature on two-dimensional flows to guess the location of coherent structures (see Section 1 for references).

3. Sufficient vs. necessary conditions. Theorem 1 provides a sufficient condition for the existence of hyperbolic material lines. It aims to locate coherent structures whose "bulk" deformation rate is slower than typical nearby Lagrangian particle speeds. As a result, the conditions of the theorem will not be satisfied by finite-time hyperbolic material lines or surfaces whose deformation rate is comparable to Lagrangian particle speeds. However, while such structures can undoubtedly be constructed in mathematical examples, typical coherent structures in fluid mechanics seem to display a strong enough separation of Eulerian and Lagrangian time scales such that they, as a rule, satisfy conditions of the type given in our theorem. This has been demonstrated in numerical studies on analogous conditions for two-dimensional turbulence (cf. [21,36]), and will also become transparent in our numerical study in Section 5.

4. Two-dimensional flows. A simplification arises in flows with a translational or rotational symmetry. In such cases one of the eigenvalues of $\nabla \mathbf{u}(\mathbf{x}(t), t)$ is always zero, and hence only statements (5) and (6) of the theorem apply with the choice $\lambda_{2 \max }^{+}=\lambda_{2 \max }^{-}=0$. Then (11) and (12) simplify to

$$
\lambda_{3 \min }>\frac{4 \beta}{5-\sqrt{17}}, \quad \lambda_{1 \min }>\frac{4 \beta}{5-\sqrt{17}},
$$

respectively. The attracting and repelling material surfaces are equivariant with respect to the underlying symmetry. If, in addition, the flow is incompressible, we have

$$
\lambda_{3 \min }=\lambda_{1 \min } \stackrel{\text { def }}{=} \lambda_{\min },
$$

and the above inequalities simplify further to the single condition

$$
\lambda_{\min }>\frac{4 \beta}{5-\sqrt{17}} .
$$

At the same time, the single condition derived in [21] for two-dimensional flows with $\mathbf{M} \in \mathbb{R}^{2 \times 2}$ is of the form

$$
\lambda_{\min }>(2+\sqrt{2}) \frac{\tilde{\beta}}{\alpha}
$$


with

$$
\tilde{\beta}=\max _{t \in \mathcal{I}}|\dot{\mathbf{M}}(t)| \equiv \max _{t \in \mathcal{I}} \sqrt{\sum_{i, j=1}^{2}\left|\dot{M}_{i j}(t)\right|^{2}}, \quad \alpha=\min _{t \in \mathcal{I}}|\operatorname{det} M(t)| .
$$

Since

$$
\beta=\max _{t \in \mathcal{I}}\left\|\mathbf{M}^{-1}(t) \dot{\mathbf{M}}(t)\right\| \leq \max _{t \in \mathcal{I}}\left|\mathbf{M}^{-1}(t) \dot{\mathbf{M}}(t)\right| \leq \frac{\sqrt{2}}{\alpha} \tilde{\beta}
$$

(14) implies the condition

$$
\lambda_{\min }>(1+\sqrt{2}) \beta
$$

for finite-time hyperbolicity of the underlying fluid particle over the time interval $\mathcal{I}$. This last condition is somewhat less restrictive than (13), i.e., allows larger derivatives for the eigenvector matrix $\mathbf{M}(t)$. The reason is that the special two-dimensional estimates used in [21] were sharper than the general, three-dimensional estimates of this paper.

5. Steady flows. The quantities $\lambda_{j}(t)$ and $\mathbf{M}(t)$ typically have a general time dependence even for steady flows, therefore our criteria do not admit any obvious simplification in the steady case.

6. Differentiability in time. Our basic assumptions require the velocity field $\mathbf{u}(\mathbf{x}, t)$ to be differentiable both in space and time. While such an assumption is questionable for a truly turbulent velocity field, our main concern here is the analysis of numerical or experimental data sets. Such data sets are routinely interpolated smoothly for the purposes of analysis, in which case their differentiability is automatically guaranteed. Naturally, one might lose small scale structures in this fashion, but our interest here lies in medium-to-large scale coherent structures that are strong enough to persist in the smoothed data set.

7. Alignment properties of vorticity. For inviscid flows material lines and vortex lines coincide, and hence the stability of vortex lines and vortex tubes can be inferred from Theorem 1. In particular, the much studied question of alignment of vorticity with eigenvectors of the rate-of-strain tensor (i.e., the symmetric part of $\nabla \mathbf{u}$ ) can be approached in a dynamically exact way. Several studies tried to find a relationship between vorticity and the rate-of-strain eigenvalues using short-term expansions derived from the instantaneous velocity field (see, e.g., $[6,13,32])$. One can, however, view any preferential alignment of vorticity as an effect due to the presence of stable material lines or material surfaces. In particular, in strain-dominated regions material curves as well as vorticity will line up with the tangent spaces of the most robust attracting material lines and surfaces. These tangent spaces are close to appropriate subspaces spanned by the eigenvectors of $\nabla \mathbf{u}$ as opposed to the eigenvectors of the rate-of-strain tensor. Our estimates in the proof of Theorem 1 (cf. Appendix A) in fact quantify this closeness in terms of the norm of the tangent of an angle which turns out to be bounded by $L(0)$ or $M(0)$ (cf. (A.23) and (A.36)). A more detailed dynamical approach to vorticity alignment is the subject of current research.

\section{Numerical implementation of Theorem 1}

\subsection{Simplified analytic conditions}

Related numerical work on two-dimensional turbulence suggests that the conditions of Theorem 1 can actually be relaxed. In particular, conditions (7)-(12) can, in practice, be replaced by milder conditions for the existence 
of smooth sets of fluid particles that do not separate from $\mathbf{x}(t)$ at the rate suggested by an instantaneous local linearization near $\mathbf{x}(t) .{ }^{3}$ In a numerical algorithm one can then replace the conditions (7) and (8) by (cf. (A.13))

$$
\beta\left(\frac{1}{\lambda_{2 \min }}+\frac{1}{\lambda_{3 \min }}\right)<1
$$

conditions (9) and (10) by (cf. (A.13))

$$
\beta\left(\frac{1}{\lambda_{2 \min }}+\frac{1}{\lambda_{1 \min }}\right)<1
$$

condition (11) by (cf. (A.30))

$$
\frac{4 \beta}{\lambda_{3 \min }-\lambda_{2 \max }^{+}}<1,
$$

condition (12) by (cf. (A.30))

$$
\frac{4 \beta}{\lambda_{3 \min }-\lambda_{2 \max }^{-}}<1,
$$

and still find distinguished material lines and surfaces with slightly milder stability properties. Using these milder formulae will reduce computation time, and typically yield further points on coherent structures that would otherwise be lost due to numerical errors.

If, finally, the velocity field $\mathbf{u}$ is two-dimensional, then the single relevant condition for the existence of finite-time hyperbolic material lines is

$$
\lambda_{\min }>\frac{4 \beta}{5-\sqrt{17}},
$$

as follows from our Remark 4 in Section 3.3.

\subsection{Hyperbolicity times and their computation}

First, we note that a practical numerical evaluation of the conditions we discussed above will yield different results in forward and backward time. A forward time verification of the relaxed hyperbolicity conditions (15)-(18) will yield unstable coherent structures, while a backward time calculation leads to stable coherent structures. The reason is that unstable boundaries are finite-time stable manifolds to distinguished trajectories, and hence points close to them will spend longer times in hyperbolic regions. At the same time, stable coherent structures are finite-time unstable manifolds that become "visible" in backward time for the same reason.

Since the time direction of the analysis immediately excludes certain types of structures from detection, it is enough to compute the following five types of hyperbolicity times for each element of a grid of particles:

\footnotetext{
${ }^{3}$ Technically, these are the conditions in our proof in Appendix A that ensure the contracting nature of the map $\mathcal{F}$, without requiring uniform attractivity for the resulting material line or surface over the interval $\mathcal{I}$.
} 


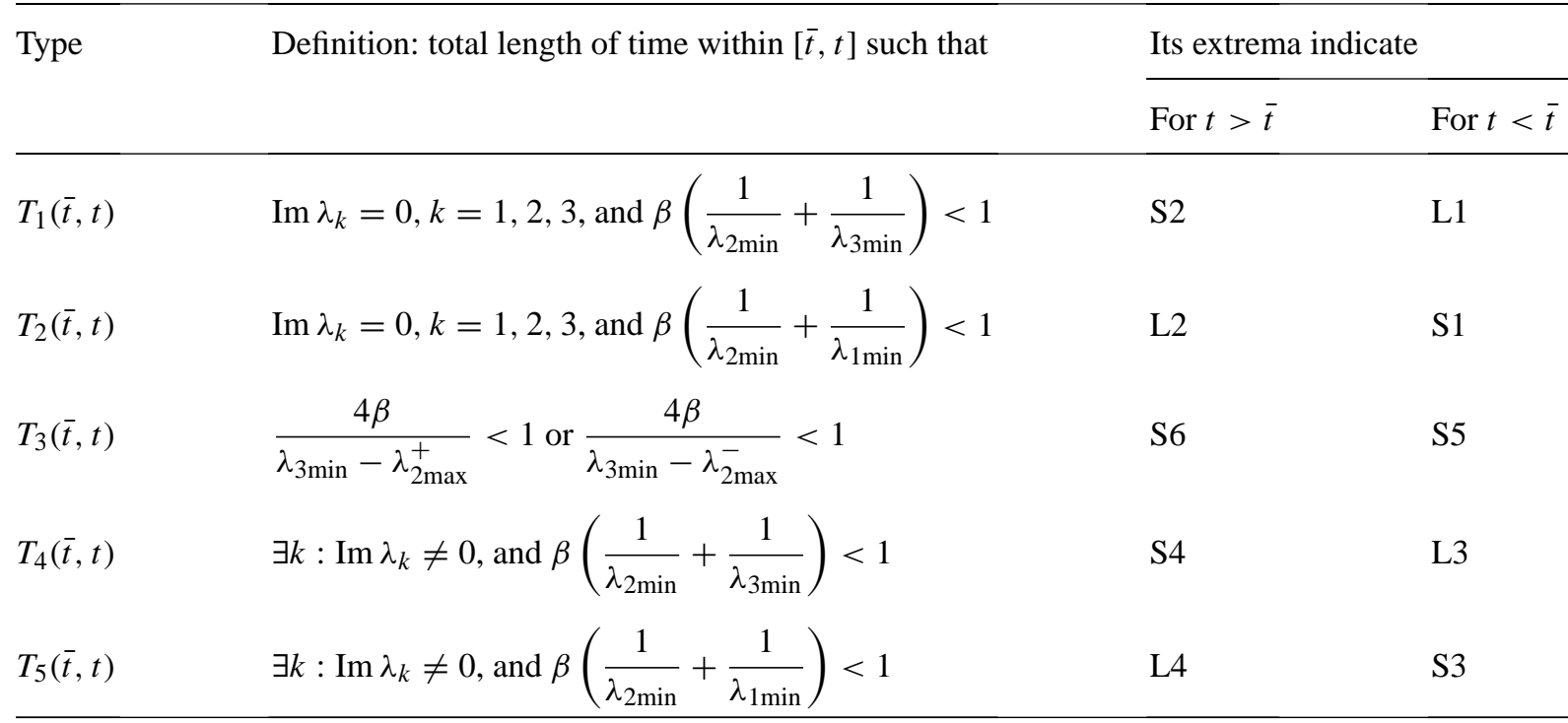

We note that for two-dimensional flows and for three-dimensional flows with a rotational, translation or helical symmetry only $T_{3}(\bar{t}, t)$ can be nonzero, since for such flows one of the eigenvalues is always zero (which also implies $\left.\lambda_{2 \max }^{+}=\lambda_{2 \max }^{-} \equiv 0\right)$.

Finally, the employment of two additional techniques appear to result in sharper numerical images:

1. Lumping the hyperbolicity times $T_{1}$ and $T_{2}$, as well as $T_{4}$ and $T_{5}$, in the same category. In this fashion, one only keeps track of two (combined) hyperbolicity times, $T_{6}$ and $T_{7}$ :

Type Definition: total length of time within $[\bar{t}, t]$ such that

Its extrema indicate

For $t>\bar{t} \quad$ For $t<\bar{t}$

$T_{6}(\bar{t}, t) \quad \forall k: \operatorname{Im} \lambda_{k}=0$, and $\beta\left(\frac{1}{\lambda_{2 \min }}+\frac{1}{\lambda_{3 \min }}\right)<1$ or $\beta\left(\frac{1}{\lambda_{2 \min }}+\frac{1}{\lambda_{1 \min }}\right)<1 \quad$ S2 or L2 $\quad$ S1 or L1

$T_{7}(\bar{t}, t) \quad \exists k: \operatorname{Im} \lambda_{k} \neq 0$, and $\beta\left(\frac{1}{\lambda_{2 \min }}+\frac{1}{\lambda_{3 \min }}\right)<1$ or $\beta\left(\frac{1}{\lambda_{2 \min }}+\frac{1}{\lambda_{1 \mathrm{~min}}}\right)<1 \quad$ S4 or L4 $\quad$ S3 or L3

2. Selecting a positive threshold below which $\left|\operatorname{Re} \lambda_{k}\right|$ will be considered zero.

\section{An example: coherent structures in steady and forced ABC flows}

We use a well-known three-dimensional velocity field, the ABC flow, to illustrate the power of our techniques. We shall not make use of Poincaré sections that have traditionally been used to study this spatially periodic flow (see $[12,23])$. Instead, we consider the flow defined only on a finite-time interval, and aim to extract coherent structures from it without relying on long-term iterations of trajectories.

In the second part of this section, we shall introduce a growing, aperiodic-in-time perturbation of the steady ABC flow. While the resulting system will not solve Euler's equation any more, the growing perturbation is a reasonable addition since the steady limit is known to be unstable (cf. [17,26]). In reality, perturbations to the steady limit will grow exponentially, but linear growth will suffice for the purposes of our finite-time study. 


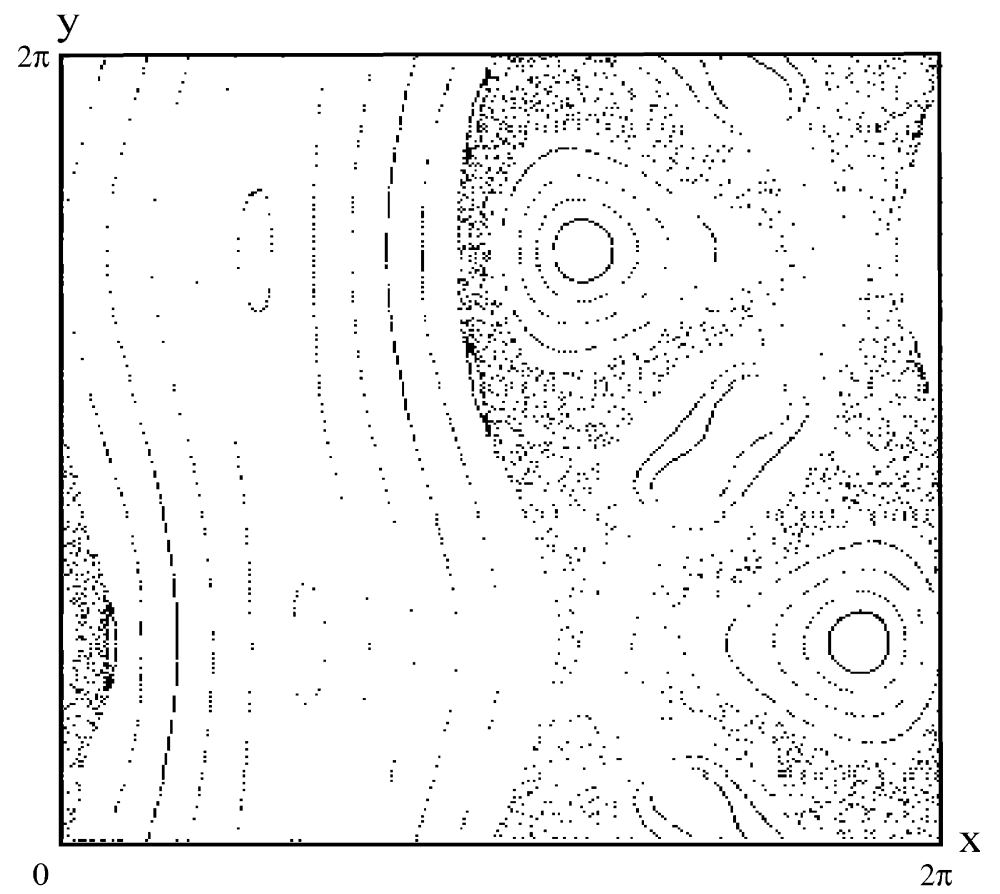

Fig. 4. Poincaré section for the $\mathrm{ABC}$ flow from Dombre et al. [12]. The parameter values are $A=1, B=\sqrt{\frac{2}{3}}, C=1 / \sqrt{3}$, and the section is taken at $z=0$.

\subsection{Steady ABC flow}

We consider the three-dimensional velocity field

$$
\dot{x}=A \sin z+C \cos y, \quad \dot{y}=B \sin x+A \cos z, \quad \dot{z}=C \sin y+B \cos x .
$$

This class of flows, known as ABC (Arnold-Beltrami-Childress) flows, is notable for being an exact steady solution of Euler's equation. It has received considerable attention as an example of a three-dimensional steady Euler flow with nontrivial streamline geometry. The complexity of streamlines is due to the fact that vorticity and velocity are everywhere parallel, resulting in the degeneracy of the Bernoulli sum, a universal first integral for three-dimensional steady Euler flows (see, e.g., [3]). It appears that the flow is nonintegrable for $A B C \neq 0$ (see [12]), a fact that was analytically confirmed for generic parameter configurations obeying $A=B$, or $A B \neq 0, A \neq B, 0<|C| \ll 1$ (see $[39,40])$.

The spatial periodicity of (19) naturally suggests the employment of Poincaré sections in numerical studies, such as that of Dombre et al. [12], who used 5000 intersections of a single trajectory with the $z=0$ plane to locate mixing regions. As shown in Fig. 4, their result gives a clear indication of the location and shape of chaotic mixing zones.

In our numerical study of this problem, we fixed the parameter values $A=\sqrt{3}, B=\sqrt{2}$, and $C=1 .{ }^{4}$ We chose a $210 \times 210$ grid of initial conditions on each face of the cube $[0,2 \pi]^{3}$ and advected these grids of particles up to time $t=10 .{ }^{5}$ In Fig. 5, we show the results of a finite-time stability analysis in forward time, based on our

\footnotetext{
${ }^{4}$ Note that up to a rescaling of time, this parameter configuration gives the same dynamics as in [12].

${ }^{5}$ By periodicity in $x, y$, and $z$, it is enough to study three different faces of the cube.
} 

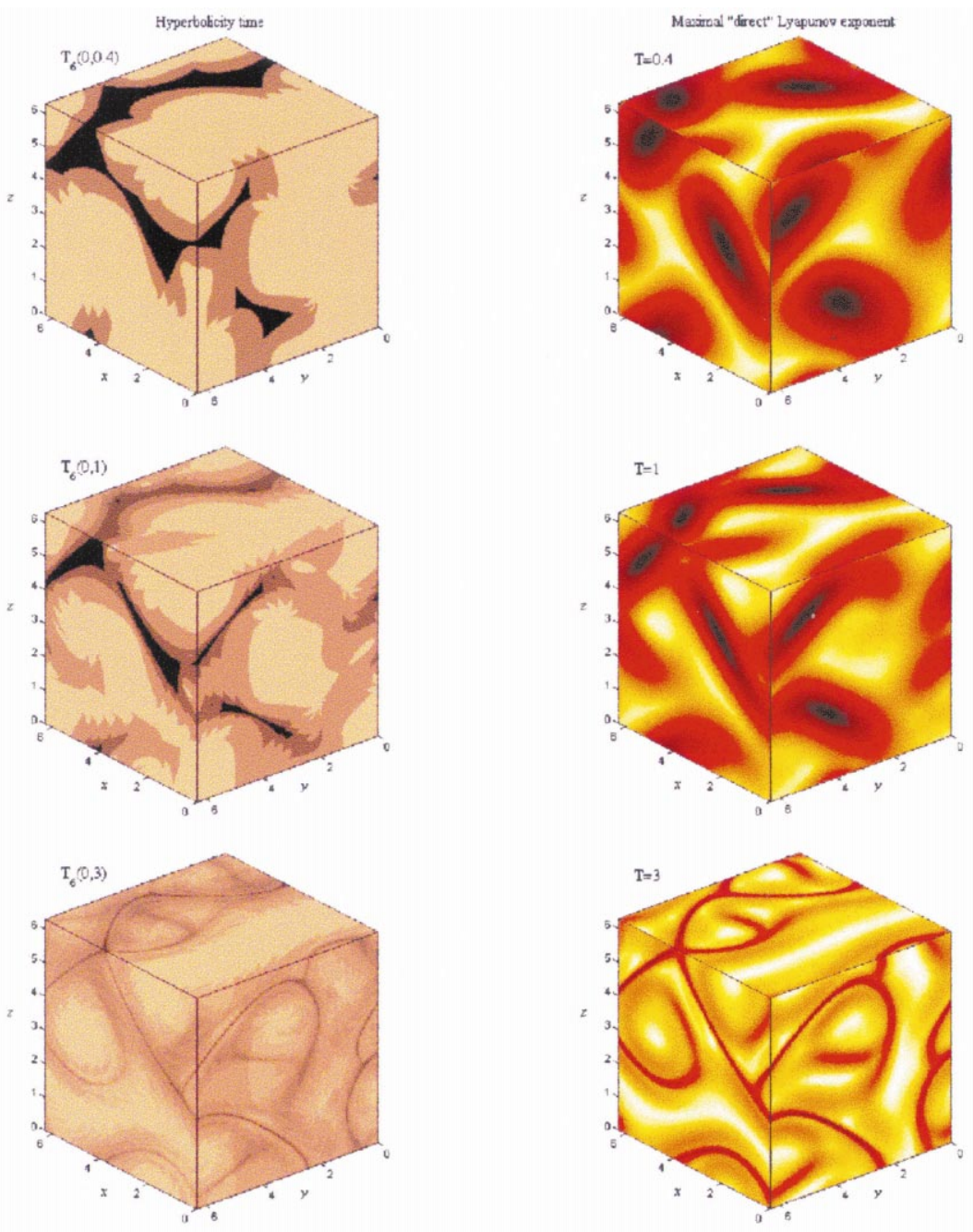

Fig. 5. Hyperbolicity times (type 6) and direct maximal Lyapunov exponents at times $t=0.4,1.0$, and 3.0 for the ABC flow. Darker colors indicate larger values. Local maxima represent repelling coherent structures at $t=0$.

discussion in Section 4. In all runs $\left|\operatorname{Re} \lambda_{i}\right|$ was considered nonzero once it reached the threshold 0.005 in order to filter out the effect of errors in the eigenvalue calculation. In the same figure, we also show the results of a direct maximal Lyapunov exponent calculation at different time instances. We recall that local maxima of these plots indicate coherent structures.

The Lagrangian coherent structures within the mixing zones (cf. Fig. 4) can easily be recognized as stable and unstable manifolds of hyperbolic periodic orbits. These periodic orbits are spatially $2 \pi$-periodic curves that intersect the faces of the cube at distinct points. Their stable and unstable manifolds are two-dimensional surfaces that intersect the faces of the cube along the curves that our techniques have located. These curves are finite and do not show the usual tangling associated with transverse homoclinic orbits. The reason is that they have been computed for finite-times. We note that our analysis confirms and extends the heuristic description of mixing given by Dombre et al. [12], who considered small perturbations of the integrable case $C=0$. 
From a computational point of view, hyperbolicity time plots converged much faster than "direct" Lyapunov exponents over short time intervals. On intermediate time scales the details of hyperbolicity time plots are more refined, but the clarity of the structures is deteriorating. One reason is that the width of coherent structures quickly decreases, due to the fast convergence of our analytic approach. As a result, the boundaries become more and more susceptible to the sizable numerical errors introduced by the low order advection scheme we used (a 4th order Runge-Kutta scheme). These errors also introduce a discrepancy between quantities associated with the velocity field and particle trajectories that grow inconsistent with the velocity field. The numerical errors are tangible since the velocity field is known to admit chaotic streamlines. As time approaches $t=10$, the plot rendered by the maximal direct Lyapunov exponents becomes very sharp, as it is not affected by the growing inconsistencies between the velocity field and individual particle motions.

Hyperbolicity plots of type 7 show similar convergence properties, but reveal further structures that are not of type 1 or 2 . We show a type 6 and type 7 plot together with the maximal direct Lyapunov exponent plot for $t=-8$ in Fig. 6. Note that the same coherent structures that are local maximizers in the type 6 plot show up as local minimizers in the type 7 plot. Also note that the type 7 plot does indicate resonant tori of hyperbolic stability type (cf. [12]), which remain hidden to the direct Lyapunov exponent calculation. The reason is the sensitivity of hyperbolicity
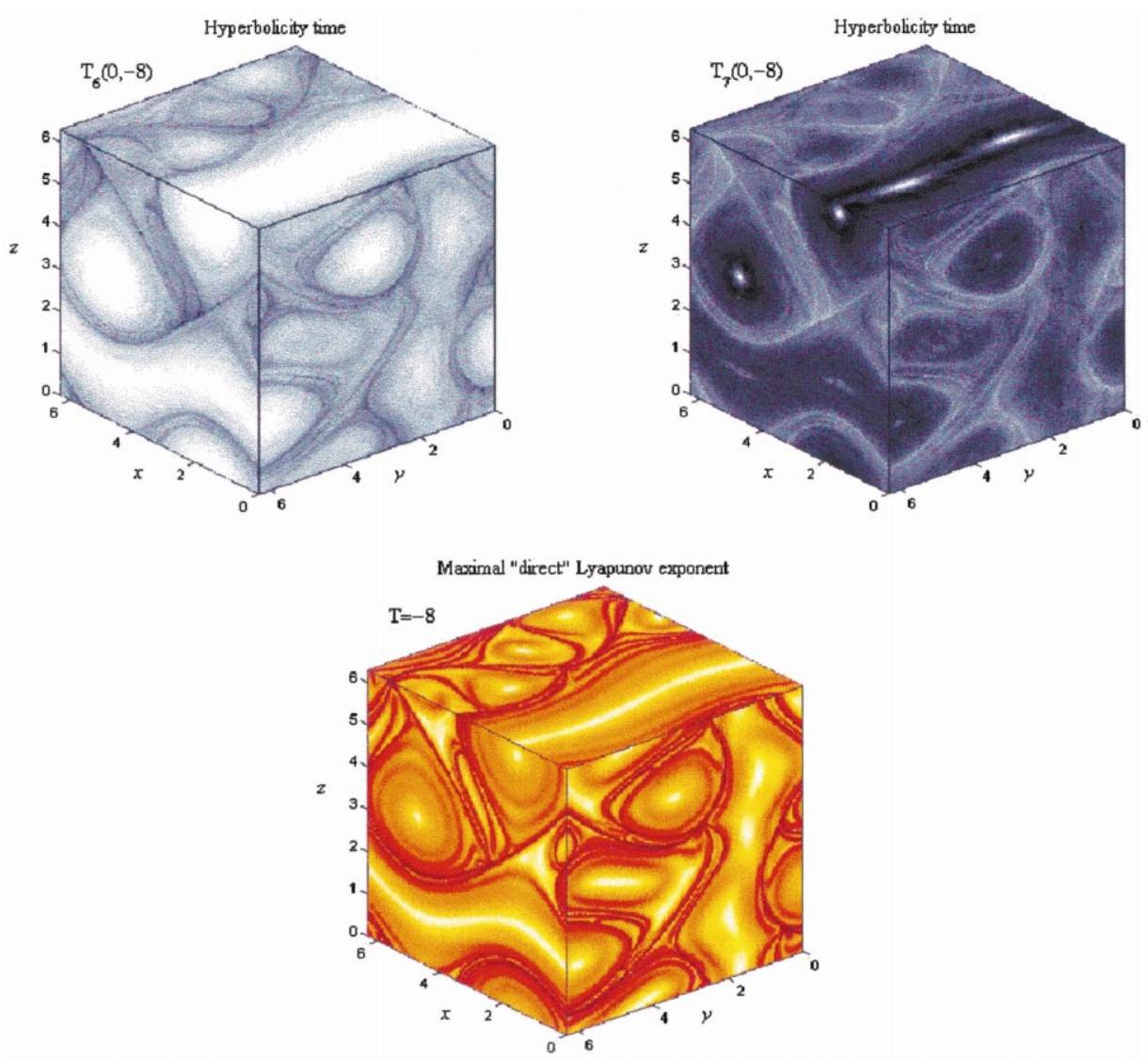

Fig. 6. Hyperbolicity time plots of type 6 and type 7, along with maximal direct Lyapunov exponents at $t=-8$ for the ABC flow. Darker colors indicate larger values. Note the appearance of resonant hyperbolic tori indicated by the low values in the type 7 plot. 

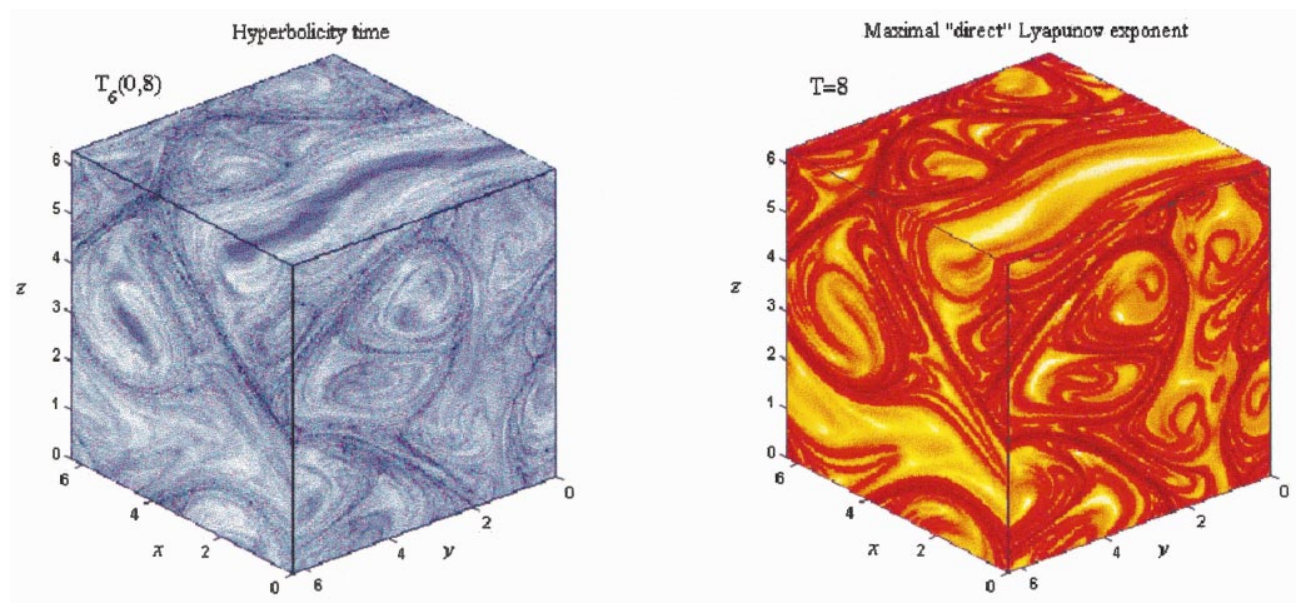

Fig. 7. Type 6 hyperbolicity time plot and direct maximal Lyapunov exponent plot for the unsteady ABC-type flow. The calculation was performed in backward time on the interval $[-8,0]$. Local maxima in the plots correspond to repelling coherent structures.

time plots to the exact stability type of orbits. In contrast, maximal Lyapunov exponent calculations merely indicate regions with different magnitudes of stretching.

We finally note that our methods do not give information about details of KAM-type surfaces, as trajectories traveling in such surfaces are only hyperbolic for short times. As a result, these surfaces fill light cylindrical regions in most of our plots. The exception is the type 7 plot shown in Fig. 6, where KAM surfaces are darker and Lagrangian coherent structures, including resonance zones, are lighter. The reason is that trajectories on KAM tori periodically revisit regions of S3 hyperbolicity type (cf. Fig. 3), while those confined to coherent structures do not.

\subsection{Unsteady ABC-type flow}

In this section, we consider an unsteady version of the $\mathrm{ABC}$ flow, given by

$$
\dot{x}=\left(A+\frac{1}{2} t \sin \pi t\right) \sin z+C \cos y, \quad \dot{y}=B \sin x+\left(A+\frac{1}{2} t \sin \pi t\right) \cos z, \quad \dot{z}=C \sin y+B \cos x .
$$

Note that this flow is still divergence free, but the amplitude $A$ is now replaced by a time-dependent term that exhibits growing oscillations. We fix the same parameter values as in the steady case. The aperiodic-in-time behavior now explicitly prevents one from applying Poincaré sections in the exploration of Lagrangian coherent structures. These structures are now time-dependent, and hence their $t=0$ snapshot will depend on the length of time used in our analysis.

We show the result of a forward time hyperbolicity time calculation along with a maximal direct Lyapunov exponent calculation in Fig. 7. The convergence of the latter is again slower in this case, but produces fairly sharp images for larger times. The hyperbolicity time approach again shows fast initial convergence, but the sharpness of the structures is now lost faster due to larger numerical errors produced by the growing oscillations of the velocity field. Still, both approaches yield distinct coherent structures.

\section{Conclusions}

In this paper, we developed a new approach to defining and extracting Lagrangian coherent structures from three-dimensional unsteady flow fields. The structures are defined as material lines and surfaces retaining the same stability time for locally the longest time in the flow. Our main theorem provides explicit formulae for 
the calculation of these stability times in terms of the invariants of the velocity field along particle paths. These results create a strong link between Eulerian and Lagrangian approaches to coherent structures. In this sense, they provide a three-dimensional extension of the dynamically "exact" Weiss criterion given by Haller and Yuan [20] for two-dimensional turbulence. Notably, our criteria are Galilean-invariant, and hence do not rely on coordinatedependent features of the velocity field, such as the location of instantaneous stagnation points. A detailed exploration of the physical meaning of our analytic conditions still awaits, but their rough meaning is the following: they distinguish material lines and surfaces that retain their stability type for long times, and display a rotation rate of the eigenvectors of $\nabla \mathbf{u}$ that is locally the slowest in the flow (cf. the conditions of Theorem 1).

To verify the special dynamical role of the material surfaces obtained in this fashion, we also discussed a technique to localize coherent structures from known particle paths. This approach leads to a "direct" calculation of the maximal finite-time Lyapunov exponent: one simply differentiates instantaneous particle positions with respect to their initial conditions numerically to obtain an approximation for $\nabla_{\mathbf{x}_{0}} \mathbf{x}\left(t, \bar{t}, \mathbf{x}_{0}\right)$. This approach looks for important lines and surfaces as locations of maximal stretching induced by $\nabla_{\mathbf{x}_{0}} \mathbf{x}\left(t, \bar{t}, \mathbf{x}_{0}\right)$. Due to growing errors in particle advection, the long-term meaning of such a calculation is more statistical than dynamical. In fact, it should be in strong correlation with long-term relative dispersion calculations (cf. [37]), that are also meant to assess stretching rates in the flow [42]. Indeed, the recent work of Bowman [5] and Hardenberg et al. [22] show sharp maxima in two-dimensional relative dispersion calculations that are approximations of the finite-type hyperbolic material lines introduced in [20].

In our study of steady and unsteady $\mathrm{ABC}$ flows a complete agreement is found between the coherent structures obtained from the two different approaches. The hyperbolicity time based calculation converged faster, giving a sharp outline of the most influential coherent structures in just a few iterations. The direct Lyapunov exponent calculation was lagging behind initially, then displayed growing clarity. In either case, the structures revealed by our computations, to the best of our knowledge, have not been identified before as the main sources of mixing in ABC flows.

As we mentioned above, the long-term meaning of the direct maximal Lyapunov exponent calculation is an open question that deserves further study. Another question of interest would be how one could implement Theorem 1 in a more sophisticated way, to avoid losing the extremely accurate coherent structures on longer time scales. A further open question is how one could efficiently use the techniques for the study of turbulent data sets, where the result of a finite-time analysis may strongly depend on the initial time chosen. One is then forced to talk about attracting and repelling material lines, as well as coherent structures, in a more statistical sense, perhaps based on ensemble averages. The application of the techniques of this paper to truly turbulent data sets is a focus of our current research and will appear elsewhere.

\section{Acknowledgements}

I would like to thank Sean Winkler for very useful remarks on finite-time Lyapunov exponents and for bringing Ref. [11] to my attention. This research was partially supported by NSF Grant No. DMS-98-00922, ONR Grant No. N00014-93-I-0691, AFOSR Grant No. F49620-00-1-0133, and an Alfred P. Sloan Fellowship.

\section{Appendix A. Proof of Theorem 1}

\section{A.1. More precise definitions}

Let $\mathbf{F}_{\mathrm{s}}^{t}: \mathbb{R}^{3} \rightarrow \mathbb{R}^{3}$ denote the solution operator of (1), defined as $\mathbf{F}_{\bar{t}}^{t}\left(\mathbf{x}_{0}\right)=\mathbf{x}\left(t ; \bar{t}, \mathbf{x}_{0}\right)$. Note that $\mathbf{F}_{\bar{t}}^{t}$ is a class $C^{1}$ map for all $t, \bar{t} \in I$, with its derivative at $x_{0}$ denoted by $\nabla \mathbf{F}_{\bar{t}}^{t}\left(x_{0}\right)$. We shall use this derivative to describe the finite-time attractive or repelling nature of material surfaces and lines. 
For the purposes of this discussion, a material surface $\mathcal{M}(t)$, with $t \in \mathcal{I}=\left[t_{1}, t_{2}\right] \subset I$, is a one-parameter family of two-dimensional, class $C^{1}$ manifolds that satisfy $\mathbf{F}_{\bar{t}}^{t}(\mathcal{M}(\bar{t}))=\mathcal{M}(t)$ for any choice of $t, \bar{t} \in \mathcal{I}$. Similarly, a material line $\mathcal{L}(t)$ is a one-parameter family of class $C^{1}$ curves satisfying $\mathbf{F}_{\bar{t}}^{t}(\mathcal{L}(\bar{t}))=\mathcal{L}(t)$. In dynamical systems terminology, $(\mathcal{M}(t), t)$ is a three-dimensional invariant manifold and $(\mathcal{L}(t), t)$ is a two-dimensional invariant manifold in the extended phase space

$$
X=\mathbb{R}^{3} \times \mathbb{R}
$$

of the $\mathbf{x}$ and $t$ variables. At any point $(\mathbf{x}, t) \in(\mathcal{M}(t), t)$, the three-dimensional tangent space of $(\mathcal{M}(t), t)$ in $X$ will be denoted as $T_{\mathbf{x}} \mathcal{M}(t)$. Our notation will be similar for material curves.

Definition A.1. We say that a material surface $\mathcal{M}(t)$ is attracting on the time interval $\mathcal{I} \subset I$, if along any trajectory $\mathbf{x}(t) \in \mathcal{M}(t)$ there exists a constant $v>0$ and a smooth family of one-dimensional subspaces $E^{\mathrm{s}}(t)$, transverse to $T_{\mathbf{x}(t)} \mathcal{M}(t)$ in $X$, such that

$$
\nabla \mathbf{F}_{\bar{t}}^{t}(\mathbf{x}(\bar{t})) \cdot E^{\mathrm{s}}(\bar{t})=E^{\mathrm{s}}(t), \quad\left\|\left.\nabla \mathbf{F}_{\bar{t}}^{t}(\mathbf{x}(\bar{t}))\right|_{E^{\mathrm{s}}(\bar{t})}\right\| \leq \mathrm{e}^{-v(t-\bar{t})}, \quad t, \bar{t} \in \mathcal{I} .
$$

We say that $\mathcal{M}(t)$ is repelling on the time interval $\mathcal{I}$ if it is attracting in backward time.

We also give an analogous definition for attracting and repelling material lines.

Definition A.2. We say that a material line $\mathcal{L}(t)$ is attracting on the time interval $\mathcal{I} \subset I$, if along any trajectory $\mathbf{x}(t) \in \mathcal{L}(t)$ there exists a constant $v>0$ and a smooth family of two-dimensional subspaces $E^{\mathrm{s}}(t)$, transverse to $T_{\mathbf{x}(t)} \mathcal{L}(t)$ in $X$, such that

$$
\nabla \mathbf{F}_{\bar{t}}^{t}(\mathbf{x}(\bar{t})) \cdot E^{\mathrm{s}}(\bar{t})=E^{\mathrm{s}}(t), \quad\left\|\left.\nabla \mathbf{F}_{\bar{t}}^{t}(\mathbf{x}(\bar{t}))\right|_{E^{\mathrm{s}}(\bar{t})}\right\| \leq \mathrm{e}^{-v(t-\bar{t})}, \quad t, \bar{t} \in \mathcal{I} .
$$

We say that $\mathcal{L}(t)$ is repelling on the time interval $\mathcal{I}$ if it is attracting in backward time.

We show the geometry near an attracting material line schematically in Fig. 8. The figure shows how a material line $\mathcal{L}(t)$ spans a two-dimensional finite-time invariant manifold in the four-dimensional extended phase space $X$. The geometry near an attracting material surface $\mathcal{M}(t)$, viewed as a three-dimensional finite-time invariant manifold in $X$, is similar.

Finally, we define finite-time hyperbolic material lines and surfaces.

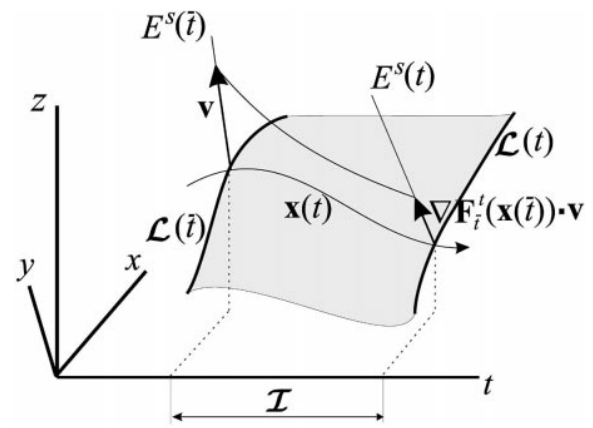

Fig. 8. Attracting material line over the time interval $\mathcal{I}$. 
Definition A.3. We say that a material surface or line is finite-time hyperbolic over the time interval $\mathcal{I}$ if it is attracting or repelling in the sense of Definitions A.1 and A.2.

A mathematically more compact definition of an attracting material surface would be that it is a finite-time invariant hypersurface in the extended phase space $X$ with a stable invariant subbundle over the time interval $I$.

The requirement for uniform attraction along trajectories in $\mathcal{M}(t)$ might sound too strong. The reason for this uniformity is that we shall ultimately want to isolate the maximal time intervals on which $\mathcal{M}(t)$ shows the same linear stability type, with no intermittent neutral behavior. Once a fixed linear stability type is assumed throughout $\mathcal{I}$, uniformity along individual trajectories holds automatically by the boundedness of our construction in time. As a result, our definitions only really require sustained linear stability for $\mathcal{M}(t)$ in order for it to qualify as an attracting material surface throughout the interval $\mathcal{I}$. A similar conclusion holds for repelling material surfaces, as well as for attracting and repelling material lines. We remind the reader of Fig. 3 which shows different types of finite-time hyperbolic material lines and surfaces.

\section{A.2. Setup for the proof}

To start the proof of statement 1 of Theorem 1, let us fix the solution $\mathbf{x}(t)$ and change variables by letting $\mathbf{y}=\mathbf{x}-\mathbf{x}(t)$. This puts the velocity field (1) in the form

$$
\dot{\mathbf{y}}=\nabla \mathbf{u}(\mathbf{x}(t), t) \mathbf{y}+\mathcal{O}(|\mathbf{y}|)^{2}
$$

with the $\mathcal{O}\left(|\mathbf{y}|^{2}\right)$ terms depending on $t$. Next we pass to a frame moving with the eigenvectors of $\nabla \mathbf{u}(\mathbf{x}(t), t)$ by letting $\mathbf{y}=\mathbf{M}(t) \mathbf{z}$, with the matrix $\mathbf{M}$ defined in (5). We obtain

$$
\dot{\mathbf{z}}=\Lambda(t) \mathbf{z}+\mathbf{f}(\mathbf{z}, t)+\mathbf{g}(\mathbf{z}, t)
$$

where

$$
\begin{aligned}
\boldsymbol{\Lambda}(t) & =\operatorname{diag}\left(\boldsymbol{\Lambda}_{\mathrm{s}}(t), \lambda_{3}(t)\right), & \boldsymbol{\Lambda}_{\mathrm{s}}(t)=\operatorname{diag}\left(\lambda_{1}(t), \lambda_{2}(t)\right), \\
\mathbf{f}(\mathbf{z}, t) & =\mathcal{O}\left(\left|\mathbf{M}^{-1}\right||\mathbf{M z}|^{2}\right), & \mathbf{g}(\mathbf{z}, t)=-\mathbf{M}^{-1} \dot{\mathbf{M}} \mathbf{z} .
\end{aligned}
$$

Using the smoothness of $\mathbf{f}$, the boundedness of $\left|\mathbf{M}^{-1}\right|$ over the time interval $\mathcal{I}$, and definition of $\beta$ in (6), we have the estimates

$$
|\mathbf{f}(\mathbf{z}, t)| \leq C_{0}|\mathbf{z}|^{2}, \quad|\mathbf{g}(\mathbf{z}, t)| \leq \beta|\mathbf{z}|
$$

with an appropriate $C_{0}>0$ for $t \in \mathcal{I}$. Note that in the $\mathbf{z}$ coordinates the original solution $\mathbf{x}(t)$ satisfies $\mathbf{z} \equiv 0$.

\section{A.3. Integral equations for a stable set in a related infinite-time system}

We want to deform the vector field (A.2) smoothly and extend its definition for all $t \in \mathbb{R}$. The objective is to turn it into a steady, linear velocity field outside a vicinity of $\mathbf{z}=0$ and outside the time interval $\mathcal{I}=\left[t_{1}, t_{2}\right]$, keeping it unchanged for small enough $|\mathbf{z}|$ and for $t$ values sufficiently separated from the boundary of $\mathcal{I}$. This can be done with the help of $C^{\infty}$ "bump functions" or mollifiers (see, e.g., [10]) as follows. For any constant $\sigma>0$, let us consider a $C^{\infty}$ function $b_{\sigma}: \mathbb{R} \rightarrow \mathbb{R}$ with the properties

$$
b_{\sigma}(s)\left\{\begin{array}{ll}
=1, & s \leq \sigma, \\
\in(0,1), & s \in(\sigma, 2 \sigma), \\
=0, & s \geq 2 \sigma,
\end{array} \quad\left|\frac{\mathrm{d}}{\mathrm{d} s} b_{\sigma}(s)\right| \leq \frac{B_{0}}{\sigma} .\right.
$$


To use this function in our construction, we select some small constants $\delta, \Delta>0$ and pass to the modified equation

$$
\dot{\mathbf{z}}=\tilde{\Lambda}(t) \mathbf{z}+\tilde{\mathbf{f}}(\mathbf{z}, t)+\tilde{\mathbf{g}}(\mathbf{z}, t),
$$

where

$$
\begin{aligned}
& \tilde{\Lambda}(t)=\Lambda(t) b_{\Delta}\left(t-\left(t_{2}-2 \Delta\right)\right) b_{\Delta}\left(\left(t_{1}+2 \Delta\right)-t\right)+\left(\begin{array}{ccc}
\lambda_{1}\left(t_{2}\right) & 0 & 0 \\
0 & \lambda_{2}\left(t_{2}\right) & 0 \\
0 & 0 & \lambda_{3}\left(t_{2}\right)
\end{array}\right) b_{\Delta}\left(\left(t_{2}+\Delta\right)-t\right) \\
& +\left(\begin{array}{ccc}
\lambda_{1}\left(t_{1}\right) & 0 & 0 \\
0 & \lambda_{2}\left(t_{1}\right) & 0 \\
0 & 0 & \lambda_{3}\left(t_{1}\right)
\end{array}\right) b_{\Delta}\left(t-\left(t_{1}-\Delta\right)\right) \\
& \tilde{\mathbf{f}}(\mathbf{z}, t)=\mathbf{f}(\mathbf{z}, t) b_{\delta}(|\mathbf{z}|) b_{\Delta}\left(t-\left(t_{2}-2 \Delta\right)\right) b_{\Delta}\left(\left(t_{1}+2 \Delta\right)-t\right), \\
& \tilde{\mathbf{g}}(\mathbf{z}, t)=\mathbf{g}(\mathbf{z}, t) b_{\Delta}\left(t-\left(t_{2}-2 \Delta\right)\right) b_{\Delta}\left(\left(t_{1}+2 \Delta\right)-t\right) .
\end{aligned}
$$

Note that the modified velocity field (A.3) is smooth for all $\mathbf{z} \in \mathbb{R}^{2}, t \in \mathbb{R}$, and coincides with (A.2) for $|\mathbf{z}| \leq \delta$ and $t \in\left[t_{1}+\Delta, t_{2}-\Delta\right]$. However, for $t \notin \mathcal{I}$, the nonlinear terms in $\tilde{\mathbf{f}}$ and the linear terms in $\tilde{\mathbf{g}}$ vanish, and $\tilde{\Lambda}(t)$ becomes time independent. The function $\tilde{\mathbf{f}}$ also vanishes for $|\mathbf{z}| \geq 2 \delta$. As a result, for all $\mathbf{z} \in \mathbb{R}^{2}$ and $t \in \mathbb{R}$, the following global estimates hold (cf. assumption (A1)):

$$
\begin{aligned}
& \tilde{\Lambda}_{11}(t) \leq-\lambda_{1 \min }<0, \quad \tilde{\Lambda}_{22}(t) \leq-\lambda_{2 \min }<0, \quad \tilde{\Lambda}_{33}(t) \geq \lambda_{3 \min }>0, \quad \tilde{\Lambda}_{j k}(t) \equiv 0, \quad j \neq k, \\
& |\tilde{\mathbf{f}}(\mathbf{z}, t)| \leq 2 C_{0} \delta|\mathbf{z}|, \quad|\tilde{\mathbf{g}}(\mathbf{z}, t)| \leq \beta|\mathbf{z}|, \quad|\nabla \tilde{\mathbf{f}}(\mathbf{z}, t)| \leq\left(C_{1}+2 B_{0} C_{0}\right) \delta, \quad|\nabla \tilde{\mathbf{g}}(\mathbf{z}, t)| \leq \beta .
\end{aligned}
$$

Introducing the notation

$$
\mathbf{z}=\left(\mathbf{z}_{\mathrm{s}}, z_{\mathrm{u}}\right) \in \mathbb{R}^{2} \times \mathbb{R}, \quad \tilde{\mathbf{f}}=\left(\mathbf{f}_{\mathrm{s}}, f_{\mathrm{u}}\right), \quad \tilde{\mathbf{g}}=\left(\mathbf{g}_{\mathrm{s}}, g_{\mathrm{u}}\right), \quad \boldsymbol{\Lambda}_{\mathrm{s}}=\left(\begin{array}{cc}
\tilde{\Lambda}_{11} & 0 \\
0 & \tilde{\Lambda}_{22}
\end{array}\right), \quad \lambda_{\mathrm{u}}=\tilde{\Lambda}_{33},
$$

we obtain from (A.3) the equivalent integral equations

$$
\begin{aligned}
& \mathbf{z}_{\mathrm{s}}(t)=\mathrm{e}^{\int_{t_{\mathrm{s}}}^{t} \boldsymbol{\Lambda}_{\mathrm{s}}(\tau) \mathrm{d} \tau} \mathbf{z}_{\mathrm{s}}\left(t_{\mathrm{S}}\right)+\int_{t_{\mathrm{s}}}^{t} \mathrm{e}^{\int_{\tau}^{t} \boldsymbol{\Lambda}_{\mathrm{s}}(s) \mathrm{d} s}\left[\mathbf{f}_{\mathrm{s}}(\mathbf{z}(\tau), \tau)+\mathbf{g}_{\mathrm{s}}(\mathbf{z}(\tau), \tau)\right] \mathrm{d} \tau, \\
& z_{\mathrm{u}}(t)=\mathrm{e}^{\int_{t_{\mathrm{u}}}^{t} \lambda_{\mathrm{u}}(\tau) \mathrm{d} \tau} z_{\mathrm{u}}\left(t_{\mathrm{u}}\right)+\int_{t_{\mathrm{u}}}^{t} \mathrm{e}^{\int_{\tau}^{t} \lambda_{\mathrm{u}}(s) \mathrm{d} s}\left[f_{\mathrm{u}}(\mathbf{z}(\tau), \tau)+g_{\mathrm{u}}(\mathbf{z}(\tau), \tau)\right] \mathrm{d} \tau .
\end{aligned}
$$

We shall first construct a stable manifold $W^{\mathrm{s}}$ to the solution $\mathbf{z}(t) \equiv 0$ of (A.6). $W^{\mathrm{s}}$ will be a three-dimensional manifold when viewed in the extended phase space of the variables $(\mathbf{z}, t)$. The subset of $W^{\mathrm{s}}$ that falls in the time interval $\left[t_{1}+\Delta, t_{2}-\Delta\right]$ will be our candidate for the repelling two-dimensional manifold $\mathcal{M}(t)$ described in statement 1 of Theorem 1 .

We fix a constant $q \in[0,1)$ (to be determined later), and define $W^{\mathrm{s}}$ as

$$
W^{\mathrm{s}}=\left\{\left(\mathbf{z}_{0}, \bar{t}\right)|\sup | \mathbf{z}\left(t ; \bar{t}, \mathbf{z}_{0}\right) \mid \mathrm{e}^{-q \lambda_{3 \min }(t-\bar{t})}<\infty\right\},
$$

where $\mathbf{z}\left(t ; \bar{t}, \mathbf{z}_{0}\right)$ is a solution of (A.6) starting from the initial condition $\mathbf{z}_{0}$ at $t=\bar{t}$. In other words, $W^{\mathrm{s}}$ contains the set of initial conditions that grow slower than $\mathrm{e}^{q \lambda_{3 \min } t}$ in forward time. Note that $W^{\mathrm{s}}$ is a positively invariant set by definition, and for the $z_{\mathrm{u}}$ component of any trajectory $(\mathbf{z}(t), t) \in W^{\mathrm{s}}$, we have

$$
\lim _{t_{\mathrm{u}} \rightarrow \infty}\left|\mathrm{e}^{\int_{t_{\mathrm{u}}}^{t} \lambda_{\mathrm{u}}(\tau) \mathrm{d} \tau} z_{\mathrm{u}}\left(t_{\mathrm{u}}\right)\right| \leq \lim _{t_{\mathrm{u}} \rightarrow \infty} \mathrm{e}^{\int_{t_{\mathrm{u}}}^{t} \lambda_{\mathrm{u}}(\tau) \mathrm{d} \tau}\left|z_{\mathrm{u}}\left(t_{\mathrm{u}}\right)\right| \leq \lim _{t_{\mathrm{u}} \rightarrow \infty} \mathrm{e}^{-\lambda_{3 \min }\left(t_{\mathrm{u}}-t\right)} K \mathrm{e}^{q \lambda_{3 \min }(t-\bar{t})}=0 .
$$


Hence, taking the limit $t_{\mathrm{u}} \rightarrow \infty$ in (A.6), we obtain that following integral equation for solutions in $W^{\mathrm{s}}$ :

$$
\begin{aligned}
& \mathbf{z}_{\mathrm{S}}(t)=\mathrm{e}^{\int_{t_{\mathrm{s}}}^{t} \boldsymbol{\Lambda}_{\mathrm{s}}(\tau) \mathrm{d} \tau} \mathbf{z}_{\mathrm{S}}\left(t_{\mathrm{S}}\right)+\int_{t_{\mathrm{s}}}^{t} \mathrm{e}^{\int_{\tau}^{t} \boldsymbol{\Lambda}_{\mathrm{s}}(s) \mathrm{d} s}\left[\mathbf{f}_{\mathrm{s}}(\mathbf{z}(\tau), \tau)+\mathbf{g}_{\mathrm{s}}(\mathbf{z}(\tau), \tau)\right] \mathrm{d} \tau, \\
& z_{\mathrm{u}}(t)=\int_{\infty}^{t} \mathrm{e}^{\int_{\tau}^{t} \lambda_{\mathrm{u}}(s) \mathrm{d} s}\left[f_{\mathrm{u}}(\mathbf{z}(\tau), \tau)+g_{\mathrm{u}}(\mathbf{z}(\tau), \tau)\right] \mathrm{d} \tau .
\end{aligned}
$$

We shall prove that under the condition given in statement 1 of the theorem, this integral equation has a unique solution $\mathbf{z}(t)$ for all $\left(\mathbf{z}_{\mathrm{s}}, t_{\mathrm{s}}\right)$, provided that $\left|\mathbf{z}_{\mathrm{s}}\right|$ is small enough. This will imply that $W^{\mathrm{s}}$ is a nonempty, two-dimensional graph over the variables $\left(\mathbf{z}_{\mathrm{s}}, t_{\mathrm{s}}\right)$.

\section{A.4. Existence of $W^{\mathrm{s}}$}

Eq. (A.8) can be recast in the form of a functional equation

$$
\mathbf{z}(t)=\mathcal{F}(\mathbf{z}(t)),
$$

which shows that, if exists, a solution of (A.8) is a fixed point of the map $\mathcal{F}$. We want to argue that $\mathcal{F}$ indeed admits a fixed point (unique for fixed $\mathbf{z}_{\mathrm{s}}$ and $t_{\mathrm{S}}$ ) when viewed as a map on an appropriate function space. Using the norm

$$
\|\mathbf{z}\|_{q}=\sup _{t \geq t_{\mathrm{s}}}|\mathbf{z}(t)| \mathrm{e}^{-q \lambda_{3 \min }\left(t-t_{\mathrm{s}}\right)},
$$

we define this function space as

$$
B_{K_{0}}^{q}=\left\{\mathbf{z}(t):\left[t_{\mathrm{s}}, \infty\right) \rightarrow \mathbb{R}^{3} \mid \mathbf{z} \in C^{0}[0, \infty),\|\mathbf{z}\|_{q} \leq K_{0}\right\}
$$

A complete metric space in the norm $\|\cdot\|_{q}, B_{K_{0}}^{q}$ contains continuous functions that grow slower in time than the expected growth of a typical solution of Eq. (A.6). We aim to show that $\mathcal{F}$ is a contraction mapping on $B_{K_{0}}^{q}$.

We start by arguing that $\mathcal{F}$ maps $B_{K_{0}}^{q}$ into itself. From Eq. (A.8) and the estimates (A.5), we obtain

$$
\begin{aligned}
|\mathcal{F}(\mathbf{z}(t))| \mathrm{e}^{-q \lambda_{3 \min }\left(t-t_{\mathrm{s}}\right) \leq} \leq & \mathrm{e}^{-q \lambda_{3 \min }\left(t-t_{\mathrm{s}}\right)}\left\{\mathrm{e}^{\int_{t_{\mathrm{s}}}^{t} \boldsymbol{\Lambda}_{\mathrm{s}}(\tau) \mathrm{d} \tau}\left|\mathbf{z}_{\mathrm{s}}\left(t_{\mathrm{s}}\right)\right|+\int_{t_{\mathrm{s}}}^{t} \mathrm{e}_{\tau}^{\int_{\tau}^{t} \boldsymbol{\Lambda}_{\mathrm{s}}(\tau) \mathrm{d} s}\left[\left|\mathbf{f}_{\mathrm{s}}(\mathbf{z}(\tau), \tau)\right|+\left|\mathbf{g}_{\mathrm{s}}(\mathbf{z}(\tau), \tau)\right|\right] \mathrm{d} \tau\right. \\
& \left.+\int_{t}^{\infty} \mathrm{e}^{\int_{\tau}^{t} \lambda_{\mathrm{u}}(s) \mathrm{d} s}\left[\left|f_{\mathrm{u}}(\mathbf{z}(\tau), \tau)\right|+\left|g_{\mathrm{u}}(\mathbf{z}(\tau), \tau)\right|\right] \mathrm{d} \tau\right\} \\
\leq & \left\|\mathrm{e}_{t_{\mathrm{s}}^{t}}^{\boldsymbol{\Lambda}_{\mathrm{s}}(\tau) \mathrm{d} \tau}\right\| \mathrm{e}^{-q \lambda_{3 \min }\left(t-t_{\mathrm{s}}\right)}\left|\mathbf{z}_{\mathrm{s}}\left(t_{\mathrm{s}}\right)\right|+\left(2 C_{0} \delta+\beta\right) \mathrm{e}^{-q \lambda_{3 \min }\left(t-t_{\mathrm{s}}\right)}\|\mathbf{z}\|_{q} \\
& \times \int_{t_{\mathrm{s}}}^{t}\left\|\mathrm{e}^{\int_{\tau}^{t} \boldsymbol{\Lambda}_{\mathrm{s}}(\tau) \mathrm{d} \tau}\right\| \mathrm{e}^{q \lambda_{3 \min }\left(\tau-t_{\mathrm{s}}\right)} \mathrm{d} \tau+\left(2 C_{0} \delta+\beta\right) \mathrm{e}^{-q \lambda_{3 \min }\left(t-t_{\mathrm{s}}\right)}\|\mathbf{z}\|_{q} \\
& \times \int_{t}^{\infty} \mathrm{e}^{\int_{\tau}^{t} \lambda_{\mathrm{u}}(s) \mathrm{d} s} \mathrm{e}^{q \lambda_{3 \min }\left(\tau-t_{\mathrm{s}}\right)} \mathrm{d} \tau
\end{aligned}
$$

From this inequality, making specific use of assumption (A1), we obtain the estimate

$$
\begin{aligned}
|\mathcal{F}(\mathbf{z}(t))| \mathrm{e}^{-q \lambda_{3 \min }\left(t-t_{\mathrm{s}}\right)} \leq & \mathrm{e}^{-\left(q \lambda_{3 \min }+\lambda_{2 \min }\right)\left(t-t_{\mathrm{s}}\right)}\left|\mathbf{z}_{\mathrm{s}}\left(t_{\mathrm{s}}\right)\right|+\left(2 C_{0} \delta+\beta\right)\|\mathbf{z}\|_{q} \mathrm{e}^{-\left(q \lambda_{3 \min }+\lambda_{2 \min }\right)\left(t-t_{\mathrm{s}}\right)} \\
& \times \int_{t_{\mathrm{s}}}^{t} \mathrm{e}^{\left(q \lambda_{3 \min }+\lambda_{2 \min }\right)\left(\tau-t_{\mathrm{s}}\right)} \mathrm{d} \tau+\left(2 C_{0} \delta+\beta\right)\|\mathbf{z}\|_{q} \mathrm{e}^{(1-q) \lambda_{3 \min }\left(t-t_{\mathrm{s}}\right)} \\
& \times \int_{t}^{\infty} \mathrm{e}^{-(1-q) \lambda_{3 \min }\left(\tau-t_{\mathrm{s}}\right)} \mathrm{d} \tau
\end{aligned}
$$


If we now restrict to initial conditions with $\left|\mathbf{z}_{\mathrm{S}}\left(t_{\mathrm{s}}\right)\right| \leq \delta$, take the suprema of both sides over $t \geq t_{\mathrm{s}}$ and use the a priori bound $\|\mathbf{z}\|_{q} \leq K_{0}$, we arrive at the inequality

$$
\|\mathcal{F}(\mathbf{z}(t))\|_{q} \leq \delta+\left(2 C_{0} \delta+\beta\right)\left(\frac{1}{q \lambda_{3 \min }+\lambda_{2 \min }}+\frac{1}{(1-q) \lambda_{3 \min }}\right) K_{0} .
$$

This shows that $\|\mathcal{F}(\mathbf{z}(t))\|_{q}<K_{0}$ will hold for small enough $\delta$ if

$$
\beta\left(\frac{1}{q \lambda_{3 \min }+\lambda_{2 \min }}+\frac{1}{(1-q) \lambda_{3 \min }}\right)<1 .
$$

It is straightforward to verify that for fixed $\beta, \lambda_{2 \min }$ and $\lambda_{3 \min }$, the left-hand side of this last expression is minimal for $q=0$. Then the least restrictive condition that we obtain from the above argument for $\mathcal{F}\left(B_{K_{0}}^{0}\right) \subset B_{K_{0}}^{0}$ is

$$
\beta\left(\frac{1}{\lambda_{2 \min }}+\frac{1}{\lambda_{3 \min }}\right)<1 .
$$

But this inequality is satisfied whenever condition (7) of the theorem holds.

For the existence of $W^{\mathrm{s}}$ it remains to show that $\mathcal{F}$ is a contraction mapping on the space $B_{K_{0}}^{0}$. For any two functions $\mathbf{z}, \hat{\mathbf{z}} \in B_{K}^{0}$ with $\mathbf{z}_{\mathrm{s}}\left(t_{\mathrm{S}}\right)=\hat{\mathbf{z}}_{\mathrm{S}}\left(t_{\mathrm{s}}\right)$, the integral equation (A.8) holds, and estimates very similar to (A.10) and (A.11) lead to

$$
\|\mathcal{F}(\mathbf{z}(t))-\mathcal{F}(\hat{\mathbf{z}}(t))\|_{0} \leq\left[\left(C_{1}+2 B_{0} C_{0}\right) \delta+\beta\right]\left(\frac{1}{\lambda_{2 \min }}+\frac{1}{\lambda_{3 \min }}\right)\|\mathbf{z}(t)-\hat{\mathbf{z}}(t)\|_{0},
$$

where we used the estimates for $|\nabla \tilde{\mathbf{f}}|$ and $|\nabla \tilde{\mathbf{g}}|$ from (A.5). Again, this last inequality will hold for $\delta>0$ small enough if (8) is satisfied. We conclude that the integral equation (A.8) has a unique solution for any $\left|\mathbf{z}_{\mathrm{s}}\right| \leq \delta$ and $t_{\mathrm{s}} \in \mathbb{R}$. As a result, for small enough $\left|\mathbf{z}_{\mathrm{s}}\right|$, the $\mathbf{z} \equiv 0$ solution of the modified system (A.3) has a unique, three-dimensional stable manifold $W^{\mathrm{s}}$, which is a graph over the variables $\mathbf{z}_{\mathrm{s}}$ and $t_{\mathrm{s}}$.

\section{A.5. Smoothness of $W^{\mathrm{s}}$}

The smoothness of $W^{\mathrm{s}}$ in $t$ follows from the smoothness of solutions of (A.10) in $t$. To establish the smoothness of $W^{\mathrm{s}}$ in $\mathbf{z}_{s 0} \equiv \mathbf{z}_{\mathrm{S}}\left(t_{\mathrm{S}}\right)$, we formally differentiate (A.8) with respect to $\mathbf{z}_{s 0}$ to obtain the following equation for $\boldsymbol{\zeta}=\left(\boldsymbol{\zeta}_{\mathrm{s}}, \boldsymbol{\zeta}_{\mathrm{u}}\right) \equiv\left(\mathrm{d} / \mathrm{d} \mathbf{z}_{s 0}\right)\left(\mathbf{z}_{\mathrm{s}}(t), z_{\mathrm{u}}(t)\right)$ :

$$
\begin{aligned}
& \zeta_{\mathrm{s}}(t)=\mathrm{e}^{\int_{t_{\mathrm{s}}}^{t} \Lambda_{\mathrm{s}}(\tau) \mathrm{d} \tau}+\int_{t_{\mathrm{s}}}^{t} \mathrm{e}^{\int_{\tau}^{t} \Lambda_{\mathrm{s}}(s) \mathrm{d} s}\left[\nabla \mathbf{f}_{\mathrm{s}}(\mathbf{z}(\tau), \tau)+\nabla \mathbf{g}_{\mathrm{s}}(\mathbf{z}(\tau), \tau)\right] \zeta(t) \mathrm{d} \tau, \\
& \zeta_{\mathrm{u}}(t)=\int_{\infty}^{t} \mathrm{e}^{\int_{\tau}^{t} \lambda_{\mathrm{u}}(s) \mathrm{d} s}\left[\nabla f_{\mathrm{u}}(\mathbf{z}(\tau), \tau)+\nabla g_{\mathrm{u}}(\mathbf{z}(\tau), \tau)\right] \zeta(t) \mathrm{d} \tau .
\end{aligned}
$$

We view this integral equation as a functional equation $\zeta=\mathcal{G}(\zeta)$, and our goal is again to establish that $\mathcal{G}$ has a unique fixed point, the actual derivative. Estimates identical to the ones derived for the existence of $W^{\mathrm{s}}$ now yield

$$
\begin{aligned}
& \|\mathcal{G}(\boldsymbol{\zeta}(t))\|_{0} \leq 1+\left[\left(C_{1}+2 B_{0} C_{0}\right) \delta+\beta\right]\left(\frac{1}{\lambda_{2 \min }}+\frac{1}{\lambda_{3 \min }}\right)\|\boldsymbol{\zeta}(t)\|_{0}, \\
& \|\mathcal{G}(\boldsymbol{\zeta}(t))-\mathcal{G}(\hat{\boldsymbol{\zeta}}(t))\|_{0} \leq\left[\left(C_{1}+2 B_{0} C_{0}\right) \delta+\beta\right]\left(\frac{1}{\lambda_{2 \min }}+\frac{1}{\lambda_{3 \min }}\right)\|\boldsymbol{\zeta}(t)-\hat{\boldsymbol{\zeta}}(t)\|_{0} .
\end{aligned}
$$

These two inequalities establish that $\mathcal{G}(\zeta(t))$ is a contraction mapping on the space

$$
B_{K_{1}}^{0}=\left\{\boldsymbol{\zeta}(t):\left[t_{\mathrm{s}}, \infty\right) \rightarrow \mathbb{R}^{3 \times 3} \mid \zeta \in C^{0}[0, \infty),\|\zeta\|_{0} \leq K_{1}\right\}
$$


for an appropriate

$$
K_{1} \geq \frac{1}{1-\left[\left(C_{1}+2 B_{0} C_{0}\right) \delta+\beta\right]\left(\left(1 / \lambda_{2 \min }\right)+\left(1 / \lambda_{3 \min }\right)\right)},
$$

and for $\delta>0$ small enough, provided that conditions (A1) and (7) hold. Consequently, the solution of the integral equation (A.8) is a class $C^{1}$ function of $\mathbf{z}_{s 0}$, i.e., for any fixed $t=t_{\mathrm{s}}, W^{\mathrm{s}} \cap\left\{t=t_{\mathrm{s}}\right\}$ is a two-dimensional, class $C^{1}$ manifold in $\mathbb{R}^{3}$.

\section{A.6. Existence and smoothness of $W^{\mathrm{u}}$}

The existence of a two-dimensional, class $C^{1}$ unstable manifold for the $\mathbf{z}(t) \equiv 0$ solution of the modified system (A.3) can be proved analogously. In particular, the existence of a unique set

$$
W^{\mathrm{u}}=\left\{\left(\mathbf{z}_{0}, \bar{t}\right)\left|\sup _{t \leq \bar{t}}\right| \mathbf{z}\left(t ; \bar{t}, \mathbf{z}_{0}\right) \mid \mathrm{e}^{q \lambda 2 \min (\bar{t}-t)}<\infty\right\},
$$

follows under the same conditions as above, for the choice $q=0$.

\section{A.7. Definition of $\mathcal{M}(t)$ and $\mathcal{L}(t)$}

For times within the interval $\left[t_{1}+\Delta, t_{2}-\Delta\right]$, we now define the material surface $\mathcal{M}(t)$ and the material line $\mathcal{L}(t)$ as

$$
\begin{aligned}
& \mathcal{M}(t)=\left\{\mathbf{z}\left|(\mathbf{z}, t) \in W^{\mathrm{s}},\right| \mathbf{z} \mid \leq \delta_{\mathrm{s}}, t \in\left[t_{1}+\Delta, t_{2}-\Delta\right]\right\} \\
& \mathcal{L}(t)=\left\{\mathbf{z}\left|(\mathbf{z}, t) \in W^{\mathrm{u}},\right| \mathbf{z} \mid \leq \delta_{\mathrm{u}}, t \in\left[t_{1}+\Delta, t_{2}-\Delta\right]\right\}
\end{aligned}
$$

Here $\delta_{\mathrm{s}}, \delta_{\mathrm{u}} \leq \delta$ are small positive constants to be selected later, and $\Delta$ is the small positive constant appearing in (A.4).

\section{A.8. $\mathcal{L}(t)$ is an attracting material line}

We now want to find conditions under which $\mathcal{L}(t)$ is an attracting material line in the sense of Definition A.2.

We start by estimating the $z_{\mathrm{u}}$ coordinate of points in $\mathcal{M}(t)$. Recall that for any fixed $t$, the surface $\mathcal{M}(t)$ is a $C^{1}$ graph over $\mathbf{z}_{\mathrm{s}}$, and hence satisfies an equation of the form

$$
z_{\mathrm{u}}=m\left(\mathbf{z}_{\mathrm{s}} ; t\right)=m^{\prime}(\mathbf{0} ; t) \cdot \mathbf{z}_{\mathrm{s}}+\mathrm{o}\left(\left|\mathbf{z}_{\mathrm{s}}\right|\right)
$$

with $m^{\prime}$ denoting the derivative of the class $C^{1}$ function $m$ with respect to $\mathbf{z}_{\mathrm{S}}$. By the integral equation (A.8), any point $\left(\hat{\mathbf{z}}_{\mathrm{s}}, \hat{z}_{\mathrm{u}}\right)$ on $\mathcal{M}(t)$ satisfies

$$
\hat{z}_{\mathrm{u}}=\int_{\infty}^{t} \mathrm{e}^{\int_{\tau}^{t} \lambda_{\mathrm{u}}(s) \mathrm{d} s}\left[f_{\mathrm{u}}(\hat{\mathbf{z}}(\tau), \tau)+g_{\mathrm{u}}(\hat{\mathbf{z}}(\tau), \tau)\right] \mathrm{d} \tau,
$$

where $\hat{\mathbf{z}}(t)$ denotes the solution crossing through the point $\left(\hat{\mathbf{z}}_{\mathrm{S}}, \hat{z}_{\mathrm{u}}\right)$ at time $t$. We can thus write

$$
m^{\prime}=\left.\zeta_{\mathrm{u}}\right|_{\mathbf{z}_{s 0}=\mathbf{0}}
$$


using our notation from Appendix A.5. Restricting to the second integral equation in (A.15), we obtain a refined version of the first inequality in (A.16) for $\zeta_{\mathrm{u}}$ :

$$
\left\|\boldsymbol{\zeta}_{\mathrm{u}}\right\|_{0} \leq\left[\left(C_{1}+2 B_{0} C_{0}\right) \delta+\beta\right] \frac{1}{\lambda_{3 \min }} K_{1} .
$$

Recalling that $\delta$ is an upper bound on $\left|\mathbf{z}_{s 0}\right|$, we can set $\delta=0$ in this last expression in order to estimate $\left\|\left.\boldsymbol{\zeta}_{\mathrm{u}}\right|_{\mathbf{z}_{s 0}=\mathbf{0}}\right\|_{0}$. Then using the smallest possible $K_{1}$ from formula (A.17) with $\delta=0$, we obtain

$$
\left|m^{\prime}\right| \leq\left\|\left.\boldsymbol{\zeta}_{\mathrm{u}}\right|_{\mathbf{z}_{50}=\mathbf{0}}\right\|_{0} \leq \frac{\beta}{\lambda_{3 \min }\left[1-\beta\left(\left(1 / \lambda_{2 \min }\right)+\left(1 / \lambda_{3 \min }\right)\right)\right]},
$$

which by (A.20) and (A.21) implies the estimate

$$
\left|z_{\mathrm{u}}\right| \leq L(\delta)\left|\mathbf{z}_{\mathrm{s}}\right|
$$

with

$$
L(\delta)=\frac{\beta+\mathrm{o}(\delta)}{\lambda_{3 \min }\left[1-\beta\left(\left(1 / \lambda_{2 \min }\right)+\left(1 / \lambda_{3 \min }\right)\right)\right]}
$$

for points in $\mathcal{M}(t)$.

With the above result at hand, we can now estimate the rate at which initial conditions approach $\mathbf{z}=0$ along the material surface $\mathcal{M}(t)$. For points on $\mathcal{M}(t)$, the first integral equation in (A.8) gives

$$
\left|\mathbf{z}_{\mathrm{S}}(t)\right| \leq \mathrm{e}^{-\lambda_{2 \min }\left(t-t_{\mathrm{s}}\right)}\left|\mathbf{z}_{s 0}\right|+\int_{t_{\mathrm{s}}}^{t} \mathrm{e}^{-\lambda_{2 \min }(t-\tau)}\left(2 C_{0} \delta+\beta\right)\left[\left|\mathbf{z}_{\mathrm{S}}(\tau)\right|+\left|z_{\mathrm{u}}(\tau)\right|\right] \mathrm{d} \tau .
$$

Introducing the notation $w(t)=\left|\mathbf{z}_{\mathrm{S}}(t)\right| \mathrm{e}^{-\lambda_{2 \min }\left(t-t_{\mathrm{S}}\right)}$ and using (A.22), we can rewrite this last inequality as

$$
w(t) \leq w\left(t_{\mathrm{s}}\right)+\int_{t_{\mathrm{s}}}^{t}\left(2 C_{0} \delta+\beta\right)(1+L(\delta)) w(\tau) \mathrm{d} \tau .
$$

Using Gronwall's inequality and then the definition of $w(t)$, we obtain

$$
\left|\mathbf{z}_{\mathrm{S}}(t)\right| \leq\left|\mathbf{z}_{\mathrm{S}}\left(t_{\mathrm{S}}\right)\right| \mathrm{e}^{-\left[\lambda_{2 \min }-\left(2 C_{0} \delta+\beta\right)(1+L(\delta))\right]\left(t-t_{\mathrm{S}}\right)} .
$$

This shows that small enough initial conditions in $\mathcal{M}(t)$ approach $\mathbf{z}=0$ at a uniform exponential rate, provided that

$$
\lambda_{2 \min }>\left(2 C_{0} \delta+\beta\right)(1+L(\delta)) .
$$

The right-hand side of this inequality is continuous in $\delta$ at $\delta=0$. As a result, it is enough to require (A.27) to hold for $\delta=0$, since then it will also hold for small enough $\delta>0$. For $\delta=0$, the inequality takes the form

$$
\lambda_{2 \min }>\beta\left[1+\frac{\beta}{\lambda_{3 \min }\left[1-\beta\left(\left(1 / \lambda_{2 \min }\right)+\left(1 / \lambda_{3 \min }\right)\right)\right]}\right] .
$$

Letting $p=\beta / \lambda_{2 \min }$ and $r=\beta / \lambda_{3 \min }$, we rewrite it as $p^{2}-2 p+1-r>0$, using the fact that $p, r>0$ and $p+r<1$ by (A.13). The solution is then easily found to be $0<p<1-\sqrt{r}$, which is equivalent to condition (7) of the theorem.

From the above argument we conclude that under assumption (A1) and condition (7), we can select $\delta_{\mathrm{s}}<\delta$ small enough in the definition of $\mathcal{M}(t)$ such that all trajectories in $\mathcal{M}(t)$ approach $\mathbf{z}=0$ at a uniform exponential rate on any time interval $\left[t_{\mathrm{s}}, t\right] \subset\left[t_{1}+\Delta, t_{2}-\Delta\right]$. As a result, there exists a class $C^{1}$ subbundle $\left(E^{\mathrm{s}}(t ; \mathbf{0}), t\right)$, the collection 
of tangent spaces of $\mathcal{M}(t)$ along the trajectory $\mathbf{z}=0$, which is invariant under the linearized flow map $\nabla F_{t_{\mathrm{s}}}^{t}(\mathbf{0})$ of Eq. (A.3), and satisfies

$$
\left\|\left.\nabla F_{t_{\mathrm{s}}}^{t}(\mathbf{0})\right|_{E^{\mathrm{s}}\left(t_{\mathrm{s}} ; \mathbf{0}\right)}\right\| \leq \mathrm{e}^{-\bar{\lambda}\left(t-t_{\mathrm{s}}\right)}
$$

for an appropriate $\bar{\lambda}>0$. Since $\bar{\lambda}$ has been constructed through a set of open conditions that involve quantities depending continuously on $\mathbf{z}$, we can in fact extend (A.28) to close enough trajectories in $\mathcal{L}(t)$. In particular, we have

$$
\left\|\left.\nabla F_{t_{\mathrm{s}}}^{t}(\mathbf{z})\right|_{E^{\mathrm{s}}\left(t_{\mathrm{s}} ; \mathbf{0}\right)}\right\| \leq \mathrm{e}^{-\left[\bar{\lambda}+\mathrm{o}\left(\delta_{\mathrm{u}}\right)\right]\left(t-t_{\mathrm{s}}\right)}, \quad \mathbf{z} \in \mathcal{L}\left(t_{\mathrm{s}}\right)
$$

for $\delta_{\mathrm{u}}$ small enough choice in the definition of $\mathcal{L}(t)$. (Note that here we rely heavily on the fact that $\mathcal{L}(t)$ is defined on a bounded time interval.) This shows that $\nabla F_{t_{\mathrm{s}}}^{t}(\mathbf{z})$ is uniformly contracting on the subbundle $\left(E^{\mathrm{s}}(t ; \mathbf{z}), t\right)$ defined as

$$
E^{\mathrm{s}}(t ; \mathbf{z}) \stackrel{\text { def }}{=} \nabla F_{t_{\mathrm{s}}}^{t}(\mathbf{z}) E^{\mathrm{s}}\left(t_{\mathrm{s}} ; \mathbf{0}\right)
$$

This proves statement 1 of Theorem 1 under assumption (A1).

\section{A.9. Proof of statement 1 under assumption (A4)}

Performing the same steps as in the case of assumption (A1), we arrive at an integral equation for $W^{\mathrm{s}}$ which is identical to (A.8). The only difference is that this time we have

$$
\boldsymbol{\Lambda}_{\mathrm{s}}=\left(\begin{array}{cc}
\tilde{\Lambda}_{11} & \tilde{\Lambda}_{12} \\
-\tilde{\Lambda}_{12} & \tilde{\Lambda}_{22}
\end{array}\right), \quad \tilde{\Lambda}_{11}(t) \equiv \tilde{\Lambda}_{22}(t) \leq-\lambda_{2 \min }<0,
$$

due to the fact that the eigenvalues $\lambda_{1}$ and $\lambda_{2}$ are now complex conjugates. However,

$$
\mathrm{e}^{\int_{t_{\mathrm{s}}}^{t} \Lambda_{\mathrm{s}}(\tau) \mathrm{d} \tau}=\mathrm{e}^{\int_{t_{\mathrm{S}}}^{t} \tilde{\Lambda}_{22}(\tau) \mathrm{d} \tau}\left(\begin{array}{cc}
\cos \int_{t_{\mathrm{s}}}^{t} \tilde{\Lambda}_{12}(\tau) \mathrm{d} \tau & -\sin \int_{t_{\mathrm{s}}}^{t} \tilde{\Lambda}_{12}(\tau) \mathrm{d} \tau \\
\sin \int_{t_{\mathrm{s}}}^{t} \tilde{\Lambda}_{12}(\tau) \mathrm{d} \tau & \cos \int_{t_{\mathrm{s}}}^{t} \tilde{\Lambda}_{12}(\tau) \mathrm{d} \tau
\end{array}\right),
$$

and hence we obtain

$$
\left\|\mathrm{e}^{\int_{t_{\mathrm{S}}}^{t} \Lambda_{\mathrm{s}}(\tau) \mathrm{d} \tau}\right\| \leq \mathrm{e}^{-\lambda_{2 \min }\left(t-t_{\mathrm{S}}\right)} .
$$

Since the exponential of $\int_{t_{\mathrm{s}}}^{t} \Lambda_{\mathrm{s}}(\tau) \mathrm{d} \tau$ obeyed the same estimate in our proof under assumption (A1), the remainder of the proof under assumption (A4) is identical to the one we gave above.

\section{A.10. Proof of statement 2}

The proof of statement 1 can be obtained by establishing the uniform attractivity of $\mathcal{M}(t)$ in backward time, in analogy with our proof for the uniform attractivity of $\mathcal{L}(t)$ in forward time. The necessary steps follow those of Appendices A.8 and A.9, with the role of the eigenvalues $\lambda_{2}$ and $\lambda_{3}$ interchanged. Accordingly, uniform attractivity in backward time follows under condition (8).

\section{A.11. Proof of statements 3 and 4}

Statements 3 and 4 can be obtained from statements 1 and 2, respectively, by reversing time and the role of $\lambda_{1}$ and $\lambda_{3}$. 


\section{A.12. Proof of statement 5}

The difficulty in this case is that the eigenvalue $\lambda_{2}(t)$ of the velocity gradient is allowed to take positive values along $\mathbf{x}(t)$, and hence exponential growth may also occur in directions tangential to the two-dimensional material surface that we want to construct. As a result, we cannot construct this surface as a stable manifold of $\mathbf{z}=0$ in system (A.3). As we shall show below, a smooth invariant manifold $W^{\mathrm{s}}$ of the type (A.7) can still be constructed if we require the instability in the direction of the eigenvector $\mathbf{e}_{3}(t)$ to dominate that in the direction of $\mathbf{e}_{2}(t)$.

We start by observing that under assumption (A3), the inequality (A.10) remains unchanged. However, instead of the resulting estimate (A.11), we now have

$$
\begin{aligned}
|\mathcal{F}(\mathbf{z}(t))| \mathrm{e}^{-q \lambda_{3 \min }\left(t-t_{\mathrm{s}}\right)} \leq & \mathrm{e}^{-\left(q \lambda_{3 \min }-\lambda_{2 \max }^{+}\right)\left(t-t_{\mathrm{s}}\right)}\left|\mathbf{z}_{\mathrm{s}}\left(t_{\mathrm{s}}\right)\right|+\left(2 C_{0} \delta+\beta\right)\|\mathbf{z}\|_{q} \mathrm{e}^{-\left(q \lambda_{3 \min }-\lambda_{2 \max }^{+}\right)\left(t-t_{\mathrm{s}}\right)} \\
& \int_{t_{\mathrm{s}}}^{t} \mathrm{e}^{\left(q \lambda_{3 \min }-\lambda_{2 \min }^{+}\right)\left(\tau-t_{\mathrm{s}}\right)} \mathrm{d} \tau+\left(2 C_{0} \delta+\beta\right)\|\mathbf{z}\|_{q} \mathrm{e}^{(1-q) \lambda_{3 \min }\left(t-t_{\mathrm{s}}\right)} \int_{t}^{\infty} \mathrm{e}^{-(1-q) \lambda_{3 \min }\left(\tau-t_{\mathrm{s}}\right)} \mathrm{d} \tau
\end{aligned}
$$

with $\lambda_{2 \max }^{+}$defined as in statement 5 of Theorem 1 . In order to avoid exponential growth in $t$ in this inequality, one is forced to select a positive $q$ for which

$$
q \lambda_{3 \min }>\lambda_{2 \max }^{+}
$$

For such a choice of $q$, we can proceed to establish the following estimates in analogy with (A.12) and (A.14):

$$
\begin{aligned}
& \|\mathcal{F}(\mathbf{z}(t))\|_{q} \leq \delta+\left(2 C_{0} \delta+\beta\right)\left(\frac{1}{q \lambda_{3 \min }-\lambda_{2 \max }^{+}}+\frac{1}{(1-q) \lambda_{3 \min }}\right) K_{0}, \\
& \|\mathcal{F}(\mathbf{z}(t))-\mathcal{F}(\hat{\mathbf{z}}(t))\|_{q} \leq\left[\left(C_{1}+2 B_{0} C_{0}\right) \delta+\beta\right]\left(\frac{1}{q \lambda_{3 \min }-\lambda_{2 \max }^{+}}+\frac{1}{(1-q) \lambda_{3 \min }}\right)\|\mathbf{z}(t)-\hat{\mathbf{z}}(t)\|_{0} .
\end{aligned}
$$

Again, these inequalities establish the existence of a unique fixed point for the map $\mathcal{F}$, provided that

$$
\beta\left(\frac{1}{q \lambda_{3 \min }-\lambda_{2 \max }^{+}}+\frac{1}{(1-q) \lambda_{3 \min }}\right)<1 .
$$

The left-hand side of this inequality attains its minimum at $q^{*}=\left(\lambda_{2 \max }^{+}+\lambda_{3 \min }\right) /\left(2 \lambda_{3 \min }\right)$, so this is the value of $q$ that we select in order to obtain the least restrictive condition for the existence of the invariant manifold $W^{\mathrm{s}}$. For $q=q^{*}$, condition (A.29) becomes

$$
\frac{4 \beta}{\lambda_{3 \min }-\lambda_{2 \max }^{+}}<1
$$

which holds whenever condition (11) of the theorem is satisfied. This completes the proof of the existence of $W^{\mathrm{s}}$. The smoothness of $W^{\mathrm{s}}$ then follows from the estimates

$$
\begin{aligned}
& \|\mathcal{G}(\boldsymbol{\zeta}(t))\|_{q^{*}} \leq 1+\left[\left(C_{1}+2 B_{0} C_{0}\right) \delta+\beta\right] \frac{4}{\lambda_{3 \min }-\lambda_{2 \max }^{+}} K_{1}, \\
& \|\mathcal{G}(\boldsymbol{\zeta}(t))-\mathcal{G}(\hat{\zeta}(t))\|_{q^{*}} \leq\left[\left(C_{1}+2 B_{0} C_{0}\right) \delta+\beta\right] \frac{4}{\lambda_{3 \min }-\lambda_{2 \max }^{+}}\|\boldsymbol{\zeta}(t)-\hat{\zeta}(t)\|_{q^{*}},
\end{aligned}
$$

in the same fashion as in the proof of statement 1 (cf. (A.16)), if we select

$$
K_{1} \geq \frac{1}{1-\left[\left(C_{1}+2 B_{0} C_{0}\right) \delta+\beta\right] 4 /\left(\lambda_{3 \min }-\lambda_{2 \max }^{+}\right)}
$$


We shall also need to use the existence of a two-dimensional strong unstable manifold $W^{\mathrm{u}}$ for the trajectory $\mathbf{z}(t)=0$ in the extended phase space $\mathbb{R}^{3} \times \mathbb{R}$. This manifold is sought as the set of points that decay to $\mathbf{z}=\mathbf{0}$ in backward time faster than $\mathrm{e}^{-q^{*} \lambda_{3 \min t}}$, i.e.,

$$
W^{\mathrm{u}}=\left\{\left(\mathbf{z}_{0}, \bar{t}\right)\left|\sup _{t \leq \bar{t}}\right| \mathbf{z}\left(t ; \bar{t}, \mathbf{z}_{0}\right) \mid \mathrm{e}^{q^{*} \lambda_{3 \min }(\bar{t}-t)}<\infty\right\} .
$$

The existence, uniqueness and smoothness of $W^{\mathrm{u}}$ follows under the same conditions as above. (Note that in this case, $W^{\mathrm{u}}$ cannot be viewed as a candidate for an attracting material curve $\mathcal{L}(t)$. The reason is that there will be instabilities transverse to $W^{\mathrm{u}}$ due to the change in the sign of $\lambda_{2}(t)$.)

In order to argue that an appropriate subset of $W^{\mathrm{s}}$ can be viewed as a repelling material surface $\mathcal{M}(t)$, we follow the strategy of Appendix A.8: we establish a backward time uniform decay estimate for small enough initial conditions in $W^{\mathrm{u}}$. This, appended to with a finite-time continuity argument, will again imply that $\mathcal{M}(t)$ is a repelling material surface for small enough $\delta_{\mathrm{s}}$ in its definition (cf. formula (A.19)).

Following the steps of Appendix A.8, we start by estimating the $\mathbf{z}_{\mathrm{s}}$ coordinate of points in $W^{\mathrm{u}}$. (While $z_{\mathrm{u}}$, as earlier, denotes a coordinate in the unstable direction associated with time-varying eigenvector $\mathbf{e}_{1}(t), \mathbf{z}_{\mathrm{S}}$ does not label purely stable directions any more; according to assumption (A3) and the assumption of statement (5) of the theorem, the sign of $\lambda_{2}$ changes within the time interval $\mathcal{I}$.) Using the definition of $W^{\mathrm{u}}$ in the integral equation (A.6), we obtain that solutions on $W^{\mathrm{u}}$ satisfy

$$
z_{\mathrm{u}}(t)=\mathrm{e}^{\int_{t_{\mathrm{u}}}^{t} \lambda_{\mathrm{u}}(\tau) \mathrm{d} \tau} z_{\mathrm{u}}\left(t_{\mathrm{u}}\right)+\int_{t_{\mathrm{u}}}^{t} \mathrm{e}_{\tau}^{\int_{\tau}^{t} \lambda_{\mathrm{u}}(s) \mathrm{d} s}\left[f_{\mathrm{u}}(\mathbf{z}(\tau), \tau)+g_{\mathrm{u}}(\mathbf{z}(\tau), \tau)\right] \mathrm{d} \tau
$$

where $t \leq t_{\mathrm{u}}$. This leads to the estimate (cf. (A.24))

$$
\left|z_{\mathrm{u}}(t)\right| \leq \mathrm{e}^{\lambda_{3 \min }\left(t-t_{\mathrm{u}}\right)}\left|\mathbf{z}_{u 0}\right|+\int_{t_{\mathrm{u}}}^{t} \mathrm{e}^{\lambda_{3 \min }(t-\tau)}\left(2 C_{0} \delta+\beta\right)\left[\left|\mathbf{z}_{\mathrm{s}}(\tau)\right|+\left|z_{\mathrm{u}}(\tau)\right|\right] \mathrm{d} \tau .
$$

The same argument that we used to establish (A.22) and (A.23) now gives

$$
\left|\mathbf{z}_{\mathrm{s}}\right| \leq M(\delta)\left|z_{\mathrm{u}}\right|
$$

with

$$
M(\delta)=\frac{2 \beta+o(\delta)}{\left(\lambda_{3 \min }-\lambda_{2 \max }^{+}\right)\left[1-\left(4 \beta /\left(\lambda_{3 \min }-\lambda_{2 \max }^{+}\right)\right)\right]}
$$

for points in the stable manifold $W^{\mathrm{s}}$. (Here we used the first inequality from (A.31) and (A.32) to obtain an estimate for (d/d $\left.z_{\mathrm{u}}\right)\left.\mathbf{z}_{\mathrm{s}}\right|_{z_{\mathrm{u}}=0}$ on $W^{\mathrm{s}}$.) Combining (A.34) and (A.35) and applying Gronwall's inequality (cf. (A.25) and (A.26)) now yields

$$
\left|z_{\mathrm{u}}(t)\right| \leq\left|z_{\mathrm{u}}\left(t_{\mathrm{u}}\right)\right| \mathrm{e}^{\left[\lambda_{3 \min }-\left(2 C_{0} \delta+\beta\right)(1+M(\delta))\right]\left(t-t_{\mathrm{s}}\right)} .
$$

As a result, small enough initial conditions in $W^{\mathrm{u}}$ will decay exponentially in backward time with a uniform exponent if

$$
\lambda_{3 \min }>\beta\left[1+\frac{2 \beta}{\left(\lambda_{3 \min }-\lambda_{2 \max }^{+}\right)\left[1-\left(4 \beta /\left(\lambda_{3 \min }-\lambda_{2 \max }^{+}\right)\right)\right]}\right] .
$$

As in Appendix A.8, we let $p=\beta / \lambda_{2 \max }^{+}$and $r=\beta / \lambda_{3 \min }$, and rewrite the above inequality as

$$
(1+2 p) r^{2}-(1+p) r+p>0,
$$


where we used the facts that by definition and by condition (A.30),

$$
0<r<p, \quad r<r^{*}(p)=\frac{p}{1+4 p} .
$$

The inequality (A.37) holds on the two semi-infinite open intervals

$$
r<\frac{1+5 p-\sqrt{(1+5 p)^{2}-4 p(1+2 p)}}{2(1+2 p)}, \quad r>\frac{1+5 p+\sqrt{(1+5 p)^{2}-4 p(1+2 p)}}{2(1+2 p)} .
$$

Since $r^{*}(p)<(1+5 p) /(2+4 p)$, only the first of these last two conditions can be satisfied. A direct calculation shows that the left-hand side of (A.37) is negative at $r^{*}(p)$ for $p>0$, hence the final solution of the system of inequalities (A.37) and (A.38) is

$$
r<\frac{1+5 p-\sqrt{(1+5 p)^{2}-4 p(1+2 p)}}{2(1+2 p)}
$$

Substituting the definition of $r$ and $p$ into this last expression yields precisely condition (11) of the theorem, thus we can conclude that initial conditions in $W^{\mathrm{s}}$ decay to $\mathbf{z}=0$ at a uniform exponential rate in backward time over the finite-time interval $\mathcal{I}$.

As in Appendix A.8, the continuity of our arguments in $\mathbf{z}_{\mathrm{s}}$ implies that $\mathcal{M}(t)$ is a repelling material surface.

\section{A.13. Proof of statement 6}

The proof of statement 6 follows that of 5 in backward time.

\section{References}

[1] H.D.I. Abarbanel, Analysis of Observed Chaotic Data, Springer, New York, 1996.

[2] P.D. Anderson, O.S. Galaktionov, G.W. Peters, F.N. Van De Vosse, H.E.H. Meijer, Analysis of mixing in three-dimensional time-periodic cavity flows, J. Fluid Mech. 386 (1999) 149-166.

[3] V.I. Arnold, B.A. Khesin, Topological Methods in Hydrodynamics, Springer, New York, 1998.

[4] K. Bajer, H.K. Moffatt, F.H. Nex, Steady confined Stokes flows with chaotic streamlines, in: H.K. Moffatt, A. Tsinober (Eds.), Topological Fluid Mechanics, Cambridge University Press, Cambridge, 1990.

[5] K.P. Bowman, Manifold geometry and mixing in observed atmospheric flows, Preprint, 1999.

[6] B.J. Cantwell, Exact solution of a restricted Euler equation for the velocity gradient tensor, Phys. Fluids A 4 (1992) 782-793.

[7] C.Q. Cheng, Y.S. Sun, Existence of invariant tori in 3D measure-preserving mappings, Cel. Mech. 47 (1990) 275-292.

[8] M.S. Chong, A.E. Perry, B.J. Cantwell, A general classification of three-dimensional flow fields, in: H.K. Moffatt, A. Tsinober (Eds.), Topological Fluid Mechanics, Cambridge University Press, Cambridge, 1990.

[9] C. Coulliette, S. Wiggins, Intergyre transport in a wind-driven, quasigeostrophic double gyre: an application of lobe dynamics, Nonlinear Proc. Geophys. 7 (2000) 59-85.

[10] A. DiBenedetto, Partial Differential Equations, Birkhäuser, Boston, 1995.

[11] R. Doerner, B. Hübinger, W. Martienssen, A. Grossmann, S. Thomae, Stable manifolds and predictability of dynamical systems, Chaos Solitons Fractals 10 (1999) 1759-1782.

[12] T. Dombre, U. Frisch, J.M. Greene, M. Hénon, A. Mehr, A.M. Soward, Chaotic streamlines in ABC flows, J. Fluid Mech. 167 (1986) 353-391.

[13] A. Dresselhaus, M. Tabor, The kinematics of stretching and alignment of material elements in general flow fields, J. Fluid Mech. 236 (1991) 415-444.

[14] M. Feingold, L.P. Kadanoff, O. Piro, Passive scalars, three-dimensional volume-preserving maps, and chaos, J. Statist. Phys. 50 (1988) $529-565$.

[15] G.O. Fountain, D.V. Khakhar, J.M. Ottino, Visualization of three-dimensional chaos, Science 281 (1998) 683-686.

[16] R.D. Frederiksen, J.A. Dahm, D.R. Dowling, Experimental assessment of fractal scale similarity in turbulent flows. Part 2. Higher-dimensional intersections and non-fractal inclusions, J. Fluid Mech. 338 (1997) 89-126.

[17] S. Friedlander, M.M. Vishik, Instability criteria for the flow of an inviscid incompressible fluid, Phys. Rev. Lett. 66 (1991) $2204-2206$. 
[18] M. Giona, A. Adrover, Invariant geometric properties of a class of 3D chaotic flows, Physica D 140 (2000) 50-68.

[19] G. Haller, A.C. Poje, Finite-time transport in aperiodic flows, Physica D 119 (1998) 352-380.

[20] G. Haller, Finding finite-time invariant manifolds in two-dimensional velocity fields, Chaos 10 (2000) 99-108.

[21] G. Haller, G. Yuan, Lagrangian coherent structures and mixing in two-dimensional turbulence, Physica D 147 (2000) 352-370.

[22] J. von Hardenberg, F. Fraedrich, F. Lunkeit, A. Provenzale, Transient chaotic mixing during a baroclinic life cycle, Chaos 10 (2000) $122-134$.

[23] M. Hénon, Sur la topologie des lignes de courant dans un cas particulier, C.R. Acad. Sci. Paris A 262 (1966) 312-314.

[24] P.J. Holmes, Some remarks on chaotic particle paths in time-periodic, 3D swirling flows, in: J.E. Marsden (Ed.), Fluids and Plasmas Geometry and Dynamics, Contemp. Math. 28 (1984) 393-401.

[25] Y.-T. Lau, J.M. Finn, Dynamics of a three-dimensional incompressible flow with stagnation points, Physica D 57 (1992) $283-310$.

[26] A. Lifschitz, Essential spectrum and local instability condition in hydrodynamics, Phys. Lett. A 152 (1991) 199-204.

[27] J.W. Loder, Y. Shen, H. Ridderinkhof, Characterization of three-dimensional Lagrangian circulation associated with tidal reflection over a submarine bank, J. Phys. Oceanogr. 27 (1997) 1729-1742.

[28] R.S. MacKay, Transport in 3D volume-preserving flows, J. Nonlinear Sci. 4 (1994) 329-354.

[29] I. Mezić, S. Wiggins, On the integrability and perturbation of three-dimensional fluid flows with symmetry, J. Nonlinear Sci. 4 (1994) 157-194.

[30] P.D. Miller, C.K.R.T. Jones, A.M. Rogerson, L.J. Pratt, Quantifying transport in numerically generated velocity fields, Physica D 110 (1997) 105-122.

[31] A.I. Neishtadt, A.A. Vasiliev, Change of the adiabatic invariant at a separatrix in a volume-preserving 3D system, Nonlinearity 12 (1999) 303-320.

[32] K.K. Nomura, G.K. Post, The structure of vorticity and rate of strain in incompressible homogeneous turbulence, J. Fluid Mech. 377 (1998) $65-97$.

[33] J.M. Ottino, The Kinematics of Mixing: Stretching, Chaos, and Transport, Cambridge University Press, Cambridge, 1989.

[34] A.M. Rogerson, P.D. Miller, L.J. Pratt, C.K.R.T. Jones, Lagrangian motion and fluid exchange in a barotropic meandering jet, J. Phys. Oceanogr. 29 (1999) 2635-2655.

[35] A.C. Poje, G. Haller, Geometry of cross-stream mixing in a double-gyre ocean model, J. Phys. Oceanogr. 29 (1999) 1649-1665.

[36] A.C. Poje, G. Haller, I. Mezić, The geometry and statistics of mixing in aperiodic flows, Phys. Fluids A 11 (1999) $2963-2968$.

[37] A. Provenzale, Transport by coherent barotropic vortices, Ann. Rev. Fluid Mech. 31 (1999) 55-93.

[38] H.A. Stone, A. Nadim, S.H. Strogatz, Chaotic streamlines inside drops immersed in steady Stokes flows, J. Fluid Mech. 232 (1991) $629-646$.

[39] S.L. Ziglin, Dichotomy of the separatrices and the nonexistence of first integrals in systems of differential equations of Hamiltonian type with two degrees of freedom, Math. USSR-Izv. 31 (1988) 407-421.

[40] S.L. Ziglin, On the nonintegrability of the ABC flow for $A=B$, Funct. Anal. Appl. 30 (1996) 80-81.

[41] J. Weiss, The dynamics of enstrophy transfer in 2-dimensional hydrodynamics, Physica D 48 (1991) 273-294.

[42] S. Winkler, Personal communication, 2000.

[43] Y.-T. Lau, J.M. Finn, Dynamics of a three-dimensional incompressible flow with stagnation points, Physica D 57 (1991) $199-204$. 